\title{
Improved Bounds for the Rate Loss of Multiresolution Source Codes
}

\author{
Hanying Feng, Member, IEEE, and Michelle Effros, Senior Member, IEEE
}

\begin{abstract}
In this paper, we present new bounds for the rate loss of multiresolution source codes (MRSCs). Considering an $M$-resolution code, the rate loss at the $i$ th resolution with distortion $D_{i}$ is defined as $L_{i}=R_{i}-R\left(D_{i}\right)$, where $R_{i}$ is the rate achievable by the MRSC at stage $i$. This rate loss describes the performance degradation of the MRSC compared to the best single-resolution code with the same distortion. For two-resolution source codes, there are three scenarios of particular interest: i) when both resolutions are equally important; ii) when the rate loss at the first resolution is $0\left(L_{1}=0\right)$; iii) when the rate loss at the second resolution is $0\left(L_{2}=0\right)$. The work of Lastras and Berger gives constant upper bounds for the rate loss of an arbitrary memoryless source in scenarios i) and ii) and an asymptotic bound for scenario iii) as $D_{2}$ approaches 0 . In this paper, we focus on the squared error distortion measure and a) prove that for scenario iii) $L_{1}<1.1610$ for all $D_{2}<D_{1}$; b) tighten the Lastras-Berger bound for scenario ii) from $L_{2} \leq 1$ to $L_{2}<0.7250$; c) tighten the Lastras-Berger bound for scenario i) from $L_{i} \leq 1 / 2$ to $L_{i}<0.3802, i \in$ $\{1,2\}$; and d) generalize the bounds for scenarios ii) and iii) to $M$-resolution codes with $M \geq 2$. We also present upper bounds for the rate losses of additive MRSCs (AMRSCs). An AMRSC is a special MRSC where each resolution describes an incremental reproduction and the $k$ th-resolution reconstruction equals the sum of the first $k$ incremental reproductions. We obtain two bounds on the rate loss of AMRSCs: one primarily good for low-rate coding and another which depends on the source entropy.
\end{abstract}

Index Terms-Additive successive refinement code, progressive transmission, tree-structured vector quantizer.

\section{INTRODUCTION}

B ECAUSE of their ability to satisfy varying bandwidth, computation, and performance constraints with a single code, multiresolution source codes (MRSCs) are playing an increasingly important role in research and in practice (e.g., [1]-[6]). A key question about MRSCs concerns the performance penalty associated with using MRSCs rather than single-resolution codes (1RSCs). In particular, for any $R_{2}>R_{1}>0$ and any $D_{1}>D_{2}>0$, we call the vector $\left(R_{1}, R_{2}, D_{1}, D_{2}\right)$ achievable by multiresolution coding on source $X$ if there exists an MRSC that uses $R_{1}$ bits per symbol

Manuscript received April 4, 2002; revised November 8, 2002. This work was supported in part by the National Science Foundation under Grant CCR9909026 and in part by the Caltech's Lee Center for Advanced Networking. The material in this paper was presented in part at the IEEE International Symposium on Information Theory (ISIT), Washington, DC, June, 2001.

H. Feng is with Teradyne, Inc., Agoura Hills, CA 91301 USA (e-mail: fhy@z.caltech.edu).

M. Effros is with the Department of Electrical Engineering (MC 136-93), California Institute of Technology, Pasadena, CA 91125 USA (e-mail: effros@z.caltech.edu).

Communicated by R. Zamir, Associate Editor for Source Coding.

Digital Object Identifier 10.1109/TIT.2003.809604

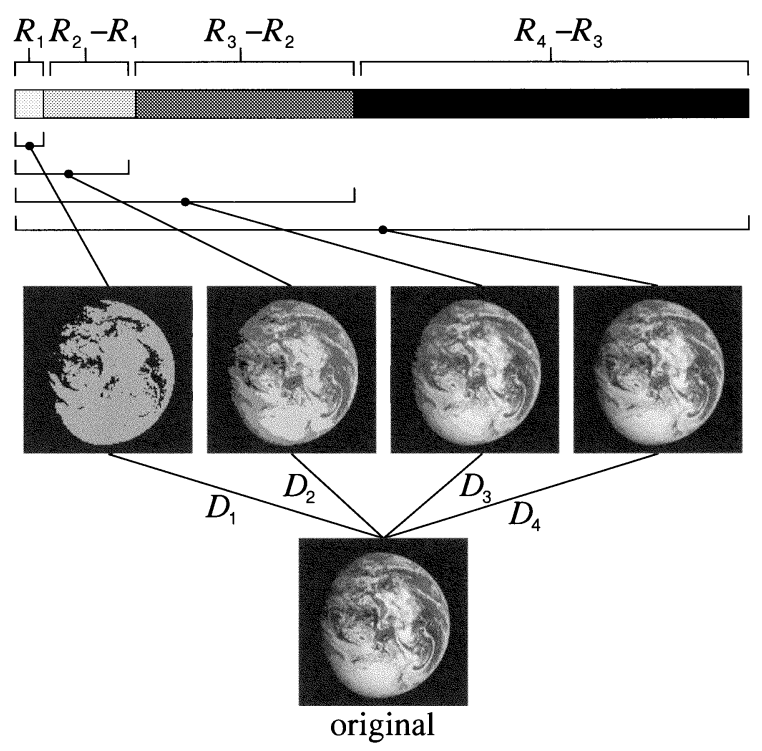

Fig. 1. A 4RSC. Decoding the first $R_{1} \mathrm{~b} / \mathrm{s}$ of the binary description yields a reproduction with distortion $D_{1}$. Decoding an additional $R_{2}-R_{1} \mathrm{~b} / \mathrm{s}$, for a total rate of $R_{2} \mathrm{~b} / \mathrm{s}$, yields a reproduction of distortion $D_{2}<D_{1}$, and so on

(b/s) to describe $X$ with distortion $D_{1}$ and then uses an additional $R_{2}-R_{1}$ b/s to refine the description to distortion $D_{2}$, as shown in Fig. 1. The rate loss of the given two-resolution code (2RSC) is defined as $L_{i}=R_{i}-R\left(D_{i}\right)(i=1,2)$, where $R(D)$ is the rate-distortion function for source $X$. Rate loss quantifies the performance degradation associated with using a 2RSC rather than the best 1RSC with the same distortion.

A source is called successively refinable if an optimal MRSC for any distortions $\left(D_{1}, D_{2}\right)$ achieves the rate-distortion bound at both stages, i.e., $L_{i}=0$ for $i=1,2$ [7]. Necessary and sufficient conditions for a source to be successively refinable appear in [8]. Examples of sources that are not successively refinable are shown for discrete-alphabet and continuous-alphabet sources in [8], [9] and [10], respectively. The MRSC achievable rate-distortion region for nonsuccessively refinable sources appears in [11] and [12].

In [13], Lastras and Berger consider the question of whether there exists a source in which the MRSC rate loss can be made arbitrarily large. Following an approach developed by Zamir in [14], they demonstrate that for any memoryless source, the squared error distortion measure, and any distortions $\left(D_{1}, D_{2}\right)$, there exists an achievable vector such that $L_{i} \leq 1 / 2$, for $i \in$ $\{1,2\}$. Moreover, they show that an achievable vector can be found with $L_{1}=0$ and $L_{2} \leq 1$. They also show that as $D_{2} \rightarrow 0$, $L_{1} \leq 1 / 2$ with $L_{2}=0$ is achievable. 
In this paper, we first present a nonasymptotic bound for $L_{1}$ when $L_{2}=0$; second, we tighten the bound for $L_{2}$ with $L_{1}=0$ from $L_{2} \leq 1$ to $L_{2}<0.7250$; third, we tighten the bound for $L_{1}=L_{2}$ from $1 / 2$ to 0.3802 ; then, we generalize the result for $L_{2}$ when $L_{1}=0$ and the result for $L_{1}$ when $L_{2}=0$ from two-resolution to $M$-resolution source codes for any $M \geq 2$.

We also consider a special type of MRSC called an additive MRSC (AMRSC). AMRSCs, also known as additive successive refinement codes, are multiple-description codes used as MRSCs. The $k$ th-resolution reproduction of an AMRSC equals the sum of the independent reconstructions from the multiple description code's first $k$ packets [15]. A two-stage AMRSC (A2RSC) encodes source $X$ using two packets with rates $R_{1}$ and $\Delta R=R_{2}-R_{1}$, respectively. The reproduction from packet 1 has expected distortion $D_{1}$, and the sum of the reproductions from both packets yields expected distortion $D_{2}<D_{1}$. AMRSCs are of potential interest since their codebook storage requirements are lower than those of other MRSCs and they provide a simple framework for low-complexity (greedy) encoding. Multistage vector quantizers are a practical example of AMRSCs. We obtain two bounds on the rate loss of AMRSCs.

\section{PRELIMINARIES}

Let $\left\{X_{i}\right\}_{i=1}^{\infty}$ be a real-valued independent and identically distributed (i.i.d.) source with probability density function (pdf) $f_{X}(x)$. Let $d$ be a real-valued nonnegative difference distortion measure, i.e., $d(x, y)=\rho(x-y)$ for any $x, y \in \boldsymbol{R}$ and some function $\rho: \boldsymbol{R} \rightarrow[0, \infty)$. Assume that $\rho$ is continuous and that there exists a reference letter $y^{*} \in \boldsymbol{R}$ such that $E_{X} d\left(X, y^{*}\right)<\infty$. For any $x^{n}, y^{n} \in \boldsymbol{R}^{n}$, define

$$
d_{n}\left(x^{n}, y^{n}\right)=\frac{1}{n} \sum_{i=1}^{n} d\left(x_{i}, y_{i}\right) .
$$

The rate-distortion function for source $\left\{X_{i}\right\}_{i=1}^{\infty}$ and the distortion measure $d$ is

$$
R(D)=\inf _{f(y \mid x): \int_{(x, y)} f(y \mid x) f_{X}(x) d(x, y) d x d y \leq D} I(X ; Y)
$$

which characterizes the minimum rate required to describe source $X$ with distortion not exceeding $D$. In the arguments that follow, we frequently assume that there exists a conditional pdf $f(y \mid x)$ that achieves $R(D)$. This assumption simplifies the exposition considerably but is not a necessary condition for any of our results.

An $\left(n, M_{1}, M_{2}\right)$ 2RSC consists of two encoder/decoder pairs: a) a coarse pair $\left(f_{n}^{(1)}, g_{n}^{(1)}\right)$

$f_{n}^{(1)}: R^{n} \rightarrow\left\{1, \ldots, M_{1}\right\} \quad$ and $\quad g_{n}^{(1)}:\left\{1, \ldots, M_{1}\right\} \rightarrow R^{n}$

with rate $(1 / n) \log M_{1}$ and distortion

$$
E d_{n}\left(X^{n}, g_{n}^{(1)}\left(f_{n}^{(1)}\left(X^{n}\right)\right)\right)
$$

and b) a refinement pair $\left(f_{n}^{(2)}, g_{n}^{(2)}\right)$

$$
f_{n}^{(2)}: \boldsymbol{R}^{n} \rightarrow\left\{1, \ldots, M_{2}\right\}
$$

and

$$
g_{n}^{(2)}:\left\{1, \ldots, M_{1}\right\} \times\left\{1, \ldots, M_{2}\right\} \rightarrow \boldsymbol{R}^{n}
$$

with total rate $(1 / n) \log \left(M_{1} M_{2}\right)$ and distortion

$$
E d_{n}\left(X^{n}, g_{n}^{(2)}\left(f_{n}^{(1)}\left(X^{n}\right), f_{n}^{(2)}\left(X^{n}\right)\right)\right) \text {. }
$$

We say that the rate-distortion vector $\left(R_{1}, R_{2}, D_{1}, D_{2}\right)$ is 2RSC-achievable if for any $\epsilon>0$ and for sufficiently large $n$, there exists an $\left(n, M_{1}, M_{2}\right) 2 \mathrm{RSC}$ such that

$$
\begin{aligned}
\frac{1}{n} \log M_{1} & \leq R_{1}+\epsilon \\
E d_{n}\left(X^{n}, g_{n}^{(1)}\left(f_{n}^{(1)}\left(X^{n}\right)\right)\right) & \leq D_{1}+\epsilon \\
\frac{1}{n} \log \left(M_{1} M_{2}\right) & \leq R_{2}+\epsilon \\
E d_{n}\left(X^{n}, g_{n}^{(2)}\left(f_{n}^{(1)}\left(X^{n}\right), f_{n}^{(2)}\left(X^{n}\right)\right)\right) & \leq D_{2}+\epsilon .
\end{aligned}
$$

The achievable region for $2 \mathrm{RSCs}$, which is defined as the set of all achievable rate-distortion vectors, is described in the following theorem. The result for finite alphabets comes from [11]. A generalization to any Polish alphabet with an escape symbol appears in [12].

Theorem 1 [11, Theorem 1], [12, Corollary 9]: For any i.i.d. source $\left\{X_{i}\right\}_{i=1}^{\infty}$ with pdf $f_{X}(x)$ and distortion measure $d$, the vector $\left(R_{1}, R_{2}, D_{1}, D_{2}\right)$ is 2 RSC achievable if there exists a conditional probability $Q_{Y_{1}, Y_{2} \mid X}$ such that

$$
\begin{array}{ll}
R_{1} \geq I\left(X ; Y_{1}\right), & E d\left(X, Y_{1}\right) \leq D_{1} \\
R_{2} \geq I\left(X ; Y_{2}, Y_{1}\right), & E d\left(X, Y_{2}\right) \leq D_{2} .
\end{array}
$$

The result generalizes to $M$ RSCs with $M>2$ [11], [12].

The definition of an $\left(n, M_{1}, M_{2}\right)$ A2RSC is similar except that the refinement decoder is defined as $g_{n}^{(2)}:\left\{1, \ldots, M_{2}\right\} \rightarrow$ $R^{n}$ and the corresponding distortion is

$$
E d_{n}\left(X^{n}, g_{n}^{(1)}\left(f_{n}^{(1)}\left(X^{n}\right)\right)+g_{n}^{(2)}\left(f_{n}^{(2)}\left(X^{n}\right)\right)\right) .
$$

The following theorem from [15] describes an achievable region for AMRSCs. ${ }^{1}$ This region is not tight. The AMRSC theorem from [15] is for discrete memoryless sources with finite alphabets. The result extends to continuous memoryless sources with an escape symbol [18].

Theorem 2 [15, Theorem 1]: For any i.i.d. source $\left\{X_{i}\right\}_{i=1}^{\infty}$ with pdf $f_{X}(x)$ and distortion measure $d,\left(R_{1}, R_{2}, D_{1}, D_{2}\right)$ is A2RSC-achievable if there exists a conditional probability $Q_{Y_{1}, Y_{2} \mid X}$ such that

$$
\begin{aligned}
R_{1} & \geq I\left(X ; Y_{1}\right), & & \operatorname{Ed}\left(X, Y_{1}\right) \leq D_{1} \\
\Delta R & \geq I(X ; \Delta Y), & & E d\left(X, Y_{2}\right) \leq D_{2}
\end{aligned}
$$

$R_{2} \geq I\left(X ; Y_{1}, \Delta Y\right)+I\left(Y_{1} ; \Delta Y\right)$ where $\Delta R=R_{2}-R_{1}$ and $Y_{1}+\Delta Y=Y_{2}$.

In this paper, we assume an arbitrary i.i.d. real-alphabet source with variance $\sigma^{2}<\infty$ and focus on the squared error distortion measure, i.e., $d(x, y)=(x-y)^{2}$. For any MRSC or AMRSC with $M$ resolutions, if rate-distortion vector $\left(R_{1}, \ldots, R_{M}, D_{1}, \ldots, D_{M}\right)$ is achievable for

$$
0<D_{M}<\cdots<D_{1} \leq \sigma^{2}
$$

\footnotetext{
${ }^{1}$ Note that the notation used here is a little different from that in [15]: $\hat{X}_{1}$ is replaced by $Y_{1}, \hat{X}_{1}+\hat{X}_{2}$ is replaced by $Y_{2}=Y_{1}+\Delta Y$, and incremental rates are replaced by total rates. The proof of this theorem, which appears in [16], is very similar to the one used by El Gamal and Cover for the multiple descriptions problem [17].
} 


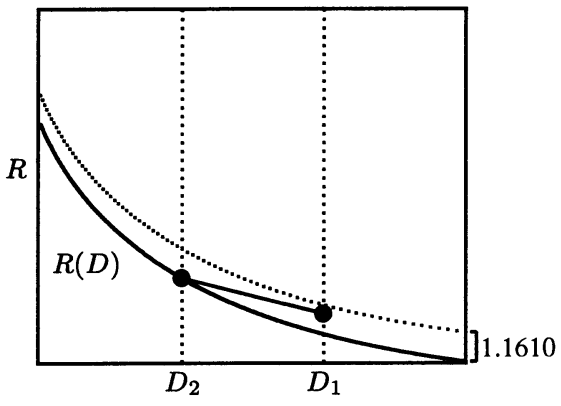

(a)

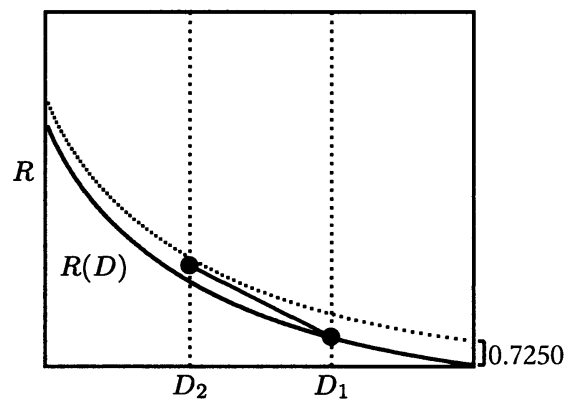

(b)

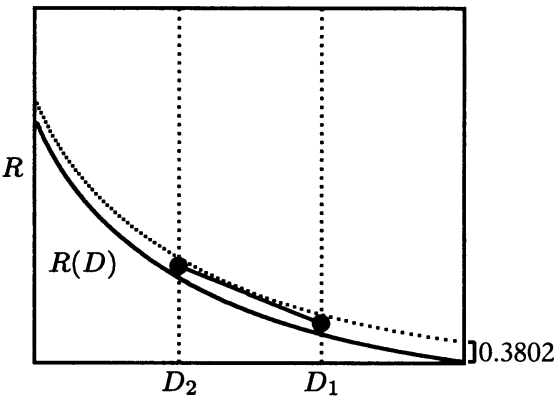

(c)

Fig. 2. Graphical interpretation of (a) Theorem 3, (b) Theorem 4, and (c) Theorem 6. In each graph, the lower curve represents $R(D)$, the upper bound represents the sum of $R(D)$ and the rate-loss bound given in the corresponding theorem, and the straight line shows the first- and second-resolution performances of a single code satisfying the corresponding bounds with equality. This approach for illustrating rate-loss bounds is the same as the one used in [13, Fig. 1].

the rate loss at the $i$ th resolution $(i \in\{1, \ldots, M\})$ is defined as $L_{i}=R_{i}-R\left(D_{i}\right) \mathrm{b} / \mathrm{s}$.

\section{TECHNIQUES}

Since Theorem 1 proves the achievability of

$$
\begin{aligned}
\left(R_{1}, R_{2},\right. & \left.D_{1}, D_{2}\right) \\
\quad & =\left(I\left(X ; Y_{1}\right), I\left(X ; Y_{1}, Y_{2}\right), \operatorname{Ed}\left(X, Y_{1}\right), \operatorname{Ed}\left(X, Y_{2}\right)\right)
\end{aligned}
$$

for any $\left(Y_{1}, Y_{2}\right)$, proving our main results involves choosing a particular $\left(Y_{1}, Y_{2}\right)$ that satisfies a desired distortion constraint $\left(D_{1}, D_{2}\right)$ and then bounding the corresponding rate losses $L_{1}=I\left(X ; Y_{1}\right)-R\left(D_{1}\right)$ and $L_{2}=I\left(X ; Y_{1}, Y_{2}\right)-R\left(D_{2}\right)$. We rely on a few simple, linear constructions (or "test channels") for $Y_{1}$ and $Y_{2}$. In particular, given some fixed distortions $D_{1}>D_{2}$, we define $U_{i}$ to be a random variable that achieves the rate-distortion function at distortion $D_{i}$. We use four main constructions in building $Y_{i}$ for source $X$

$$
\begin{array}{ll}
Y_{i}=U_{i} & Y_{i}=X+N \\
Y_{i}=(1-\alpha) X+\alpha U_{i-1}+N, & Y_{i}=U_{i+1}+N
\end{array}
$$

where $N$ is zero-mean Gaussian noise independent of $U_{i-1}, U_{i}$, $U_{i+1}$, and $X, \alpha$ is a constant, and both $\alpha$ and the variance of $N$ are chosen to satisfy the desired rate-distortion constraint. Note that the third and the fourth build a reconstruction for resolution $i$ from the rate distortion achieving reconstruction for resolution $i-1$ or $i+1$. The first two constructions were also used in [13]. Our application of both new constructions and new combinations of old constructions yields the new results. In all cases, bounding the rate loss requires bounding the difference $I\left(X ; Y_{1}, \ldots, Y_{i}\right)-I\left(X ; U_{i}\right)$. In several cases, the results given involve finding bounds associated with several choices of the vector $\left(Y_{1}, Y_{2}\right)$ and then combining them. Combinations either apply different bounds for different values of $\left(D_{1}, D_{2}\right)$ or involve convex combinations of several bounds (since the rate loss turns out to be convex, as shown in Lemma 2).

\section{RESULTS}

Throughout this section we assume an i.i.d. source $X_{1}$, $X_{2}, \ldots$ and the squared error distortion measure.

We first establish a previously unknown property of rate-distortion functions, which is used in the proof of Theorem 3. All lemmas are proved in the Appendix.
Lemma 1: Suppose $R(D)$ is the rate-distortion function of an arbitrary i.i.d. source $\left\{X_{i}\right\}_{i=1}^{\infty}$ with distortion measure $d(x, y)=(x-y)^{2}$ and $0<D_{2}<D_{1}$, then

$$
R\left(D_{2}\right)-R\left(D_{1}\right) \leq \frac{1}{2} \log \frac{D_{1}}{D_{2}}
$$

Theorem 3 bounds the first-resolution rate loss of a 2RSC achieving $R_{2}=R\left(D_{2}\right)$. Roughly, the proof involves finding a Gaussian approximation of the optimizing reproduction distribution and bounding the optimal rate loss by the rate loss of the approximation. Use of several Gaussian approximations leads to both increasing and decreasing rate loss bounds for one code, and the intersection of these bounds yields the desired constant bound on the rate loss. Fig. 2(a) shows the graphical interpretation of this theorem. Achieving performance on the rate-distortion curve in resolution 2 requires a rate penalty in resolution 1 that never exceeds $(1 / 2) \log 5$.

Theorem 3: For any $\left(D_{1}, D_{2}\right)$ with $D_{2}<D_{1}$, there exists a 2RSC-achievable rate-distortion vector $\left(R_{1}, R_{2}, D_{1}, D_{2}\right)$ with $L_{2}=0$ and $L_{1} \leq(1 / 2) \log 5 \mathrm{~b} / \mathrm{s}$.

Proof: We here design a code and derive two bounds on the rate loss. Both bounds are functions of $D_{2} / D_{1}$; one decreases as a function of $D_{2} / D_{1}$ and applies for all $D_{2} / D_{1}<1$ ( $D_{2}<D_{1}$ by assumption), while the other increases as a function of $D_{2} / D_{1}$ and applies when $D_{2} / D_{1}<1 / 2$. We combine these functions by choosing the best applicable bound for each value of $D_{2} / D_{1}$. We begin with the increasing bound.

Let $U_{1}$ and $U_{2}$ be the random variables that achieve $R\left(D_{1}\right)$ and $R\left(D_{2}\right)$, respectively, i.e., $I\left(X ; U_{i}\right)=R\left(D_{i}\right)$ and $\operatorname{Ed}\left(X, U_{i}\right) \leq D_{i}$ for $i=1,2$. Next, let $N_{1}$ be a Gaussian random variable with mean 0 and variance $D_{1}-D_{2}$ and $N_{2}$ be another Gaussian random variable with mean 0 and variance $D_{2}$ (written $N_{1} \sim \mathcal{N}\left(0, D_{1}-D_{2}\right)$ and $N_{2} \sim \mathcal{N}\left(0, D_{2}\right)$ ). Set $\left(N_{1}, N_{2}\right)$ to be independent of $\left(X, U_{1}, U_{2}\right)$ and $N_{1}$ to be independent of $N_{2}$ (written $\left(N_{1}, N_{2}\right) \Perp\left(X, U_{1}, U_{2}\right)$ and $\left.N_{1} \Perp N_{2}\right)$. Thus, $E d\left(X, X+N_{1}+N_{2}\right)=D_{1}$ and $E d\left(X, U_{2}+N_{1}\right) \leq D_{1}$. We define

$$
Y_{1}=U_{2}+N_{1} \text { and } Y_{2}=U_{2} \text {. }
$$

Here $Y_{1}$ and $Y_{2}$ satisfy the distortion constraints, i.e., $E d\left(X, Y_{2}\right) \leq D_{2}$ and $E d\left(X, Y_{1}\right) \leq D_{1}$, and, by Theorem 1, 
the vector $\left(I\left(X ; Y_{1}\right), I\left(X ; Y_{2}, Y_{1}\right), D_{1}, D_{2}\right)$ is achievable. The rate loss at the second stage is

$$
\begin{aligned}
L_{2}=I\left(X ; Y_{2}, Y_{1}\right)- & R\left(D_{2}\right) \\
& =I\left(X ; U_{2}, U_{2}+N_{1}\right)-I\left(X ; U_{2}\right)=0
\end{aligned}
$$

since $X \rightarrow U_{2} \rightarrow U_{2}+N_{1}$ forms a Markov chain. The rate loss at the first stage is

$$
\begin{aligned}
L_{1}= & I\left(X ; Y_{1}\right)-I\left(X ; U_{1}\right)=I\left(X ; U_{2}+N_{1}\right)-I\left(X ; U_{1}\right) \\
= & {\left[I\left(X ; U_{2}+N_{1}\right)-I\left(X ; X+N_{2}+N_{1}\right)\right] } \\
& +\left[I\left(X ; X+N_{2}+N_{1}\right)-I\left(X ; U_{1}\right)\right] .
\end{aligned}
$$

We bound the difference between $I\left(X ; U_{2}+N_{1}\right)$ and $I(X ; X+$ $\left.N_{2}+N_{1}\right)$ in addition to the difference between $I\left(X ; X+N_{2}+\right.$ $\left.N_{1}\right)$ and $R\left(D_{1}\right)=I\left(X ; U_{1}\right)$ to bound the rate loss. Let $L_{1 A}$ denote the first difference on the right-hand side of (1); then

$$
\begin{aligned}
L_{1 A}= & I\left(X ; U_{2}+N_{1}\right)-I\left(X ; X+N_{2}+N_{1}\right) \\
\leq & I\left(X ; U_{2}+N_{1}\right)-I\left(X ; U_{2}\right)+I\left(X ; X+N_{2}\right) \\
& -I\left(X ; X+N_{2}+N_{1}\right) \\
= & I\left(X ; U_{2}+N_{1}\right)-I\left(X ; U_{2}, U_{2}+N_{1}\right) \\
& +I\left(X ; X+N_{2}, X+N_{2}+N_{1}\right) \\
& -I\left(X ; X+N_{2}+N_{1}\right) \\
= & I\left(X ; X+N_{2} \mid X+N_{2}+N_{1}\right)-I\left(X ; U_{2} \mid U_{2}+N_{1}\right) \\
= & I\left(N_{2}+N_{1} ; N_{1} \mid X+N_{2}+N_{1}\right) \\
& -I\left(U_{2}+N_{1}-X ; N_{1} \mid U_{2}+N_{1}\right) \\
= & I\left(N_{1} ; N_{2}+N_{1}, X+N_{2}+N_{1}\right) \\
& -I\left(N_{1} ; X+N_{2}+N_{1}\right) \\
& -I\left(U_{2}+N_{1}-X ; N_{1} \mid U_{2}+N_{1}\right) \\
= & I\left(N_{1} ; N_{2}+N_{1}\right)-I\left(N_{1} ; X+N_{2}+N_{1}\right) \\
& -I\left(U_{2}+N_{1}-X ; N_{1} \mid U_{2}+N_{1}\right) \\
= & I\left(N_{1} ; N_{2}+N_{1}\right)-I\left(N_{1} ; X+N_{2}+N_{1}\right) \\
& +I\left(N_{1} ; U_{2}+N_{1}\right)-I\left(N_{1} ; U_{2}+N_{1}-X\right) \\
& -I\left(N_{1} ; U_{2}+N_{1} \mid U_{2}+N_{1}-X\right)
\end{aligned}
$$

where (2) follows since $E d\left(X, X+N_{2}\right)=D_{2}$ implies that

$$
I\left(X ; U_{2}\right)=R\left(D_{2}\right) \leq I\left(X ; X+N_{2}\right)
$$

(3) follows since $N_{1} \Perp\left(X, U_{2}, N_{2}\right)$ implies

$$
X \rightarrow U_{2} \rightarrow U_{2}+N_{1}
$$

and

$$
X \rightarrow X+N_{2} \rightarrow X+N_{2}+N_{1}
$$

form Markov chains, (4) follows since $h(A \mid B)=h(A-B \mid B)$, and (5) follows since $\left(N_{1}, N_{2}\right) \Perp X$ implies

$$
N_{1} \rightarrow N_{2}+N_{1} \rightarrow X+N_{2}+N_{1}
$$

forms a Markov chain.

Let

$$
\begin{aligned}
J & =I\left(N_{1} ; U_{2}+N_{1} \mid U_{2}+N_{1}-X\right) \\
& =I\left(U_{2}-X ; X \mid U_{2}+N_{1}-X\right)
\end{aligned}
$$

and

$$
K=I\left(N_{1} ; U_{2}+N_{1}\right)-I\left(N_{1} ; X+N_{2}+N_{1}\right)-J .
$$

Then $L_{1 A} \leq I\left(N_{1} ; N_{2}+N_{1}\right)-I\left(N_{1} ; U_{2}+N_{1}-X\right)+K$, and by the chain rule

$$
\begin{aligned}
K= & I\left(N_{1} ; U_{2}+N_{1} \mid X+N_{2}+N_{1}\right) \\
& -I\left(N_{1} ; X+N_{2}+N_{1} \mid U_{2}+N_{1}\right)-J \\
\leq & I\left(N_{1} ; U_{2}+N_{1} \mid X+N_{2}+N_{1}\right)-J \\
= & I\left(X+N_{2} ; X-U_{2}+N_{2} \mid X+N_{2}+N_{1}\right)-J \\
\leq & I\left(X+N_{2} ; X-U_{2}+N_{2}\right)-J \\
\leq & I\left(N_{2}, X+N_{2} ; X-U_{2}+N_{2}\right)-J \\
= & I\left(N_{2} ; X-U_{2}+N_{2}\right) \\
& +I\left(X-U_{2}+N_{2} ; X+N_{2} \mid N_{2}\right)-J \\
= & I\left(N_{2} ; X-U_{2}+N_{2}\right)+I\left(U_{2}-X ; X \mid N_{2}\right) \\
& -I\left(U_{2}-X ; X \mid U_{2}-X+N_{1}\right) \\
= & I\left(N_{2} ; X-U_{2}+N_{2}\right)+I\left(U_{2}-X ; X\right) \\
& -I\left(U_{2}-X ; X \mid U_{2}-X+N_{1}\right) \\
= & I\left(N_{2} ; X-U_{2}+N_{2}\right)+I\left(U_{2}-X ; U_{2}-X+N_{1}\right) \\
& -I\left(U_{2}-X ; U_{2}-X+N_{1} \mid X\right) \\
\leq & I\left(N_{2} ; X-U_{2}+N_{2}\right)+I\left(U_{2}-X ; U_{2}-X+N_{1}\right)
\end{aligned}
$$

where (6) follows since $N_{1} \Perp\left(X, U_{2}, N_{2}\right)$ implies that $X-U_{2}+N_{2} \rightarrow X+N_{2} \rightarrow X+N_{2}+N_{1}$ forms a Markov chain, (7) follows since $N_{2} \Perp\left(X, U_{2}\right)$, and (8) follows by the chain rule since

$$
\begin{aligned}
& I\left(U_{2}-X ; X, U_{2}-X+N_{1}\right) \\
&= I\left(U_{2}-X ; X\right)+I\left(U_{2}-X ; U_{2}-X+N_{1} \mid X\right) \\
&= I\left(U_{2}-X ; U_{2}-X+N_{1}\right) \\
&+I\left(U_{2}-X ; X \mid U_{2}-X+N_{1}\right) .
\end{aligned}
$$

Thus, if $2 D_{2}<D_{1}$ and we define $N_{2}^{\prime} \sim \mathcal{N}\left(0, D_{1}-2 D_{2}\right)$ and $N_{2}^{\prime} \Perp\left(X, U_{2}, N_{1}, N_{2}\right)$, then

$$
\begin{aligned}
I\left(N_{1} ;\right. & \left.U_{2}+N_{1}-X\right) \\
= & I\left(N_{2}+N_{2}^{\prime} ; X-U_{2}+N_{2}+N_{2}^{\prime}\right) \\
= & I\left(N_{2}^{\prime}, N_{2}+N_{2}^{\prime} ; X-U_{2}+N_{2}+N_{2}^{\prime}\right) \\
= & I\left(N_{2}^{\prime} ; X-U_{2}+N_{2}+N_{2}^{\prime}\right) \\
& +I\left(N_{2}+N_{2}^{\prime} ; X-U_{2}+N_{2}+N_{2}^{\prime} \mid N_{2}^{\prime}\right) \\
= & I\left(N_{2}^{\prime} ; X-U_{2}+N_{2}+N_{2}^{\prime}\right)+I\left(N_{2} ; X-U_{2}+N_{2}\right)
\end{aligned}
$$

where (9) follows since $N_{2}^{\prime} \rightarrow N_{2}+N_{2}^{\prime} \rightarrow X-U_{2}+N_{2}+N_{2}^{\prime}$ forms a Markov chain.

As a result, if $2 D_{2}<D_{1}$

$$
\begin{aligned}
L_{1 A} \leq & I\left(N_{1} ; N_{1}+N_{2}\right)+I\left(U_{2}-X ; U_{2}-X+N_{1}\right) \\
& -I\left(N_{1} ; U_{2}+N_{1}-X\right)+I\left(N_{2} ; X-U_{2}+N_{2}\right) \\
= & I\left(N_{1} ; N_{1}+N_{2}\right)+I\left(U_{2}-X ; U_{2}-X+N_{1}\right) \\
& -I\left(N_{2}^{\prime} ; X-U_{2}+N_{2}+N_{2}^{\prime}\right) \\
\leq & \frac{1}{2} \log \frac{D_{1}}{D_{2}}+\frac{1}{2} \log \frac{D_{1}}{D_{1}-D_{2}}-\frac{1}{2} \log \frac{D_{1}}{2 D_{2}} \\
= & \frac{1}{2} \log \frac{2 D_{1}}{D_{1}-D_{2}}
\end{aligned}
$$

where (10) follows from $[19$, p. 263, Problem 1] and $E(X-$ $\left.U_{2}\right)^{2} \leq D_{2}$. 
We denote the second difference on the right-hand side of (1) by $L_{1 B}$, which is bounded by $1 / 2$. The proof parallels that of [13, Theorem 3]. In particular

$$
\begin{aligned}
L_{1 B} & =I\left(X ; X+N_{2}+N_{1}\right)-I\left(X ; U_{1}\right) \\
& =I\left(X ; X+N_{2}+N_{1} \mid U_{1}\right)-I\left(X ; U_{1} \mid X+N_{2}+N_{1}\right) \\
& \leq I\left(X ; X+N_{2}+N_{1} \mid U_{1}\right) \\
& =I\left(X-U_{1} ; X-U_{1}+N_{2}+N_{1} \mid U_{1}\right) \\
& \leq I\left(X-U_{1} ; X-U_{1}+N_{2}+N_{1}\right) \\
& \leq \frac{1}{2} .
\end{aligned}
$$

Thus, if $2 D_{2}<D_{1}$, we can bound $L_{1}$ as

$$
\begin{aligned}
L_{1} & =L_{1 A}+L_{1 B} \leq \frac{1}{2} \log \frac{2 D_{1}}{D_{1}-D_{2}}+\frac{1}{2} \\
& =1+\frac{1}{2} \log \frac{D_{1}}{D_{1}-D_{2}} .
\end{aligned}
$$

We derive the second bound on $L_{1}$ by noting that for any $D_{2}<D_{1}$, the rate loss at the first stage can also be bounded as

$$
\begin{aligned}
L_{1} & =I\left(X ; Y_{1}\right)-R\left(D_{1}\right) \leq I\left(X ; Y_{2}, Y_{1}\right)-R\left(D_{1}\right) \\
& =R\left(D_{2}\right)-R\left(D_{1}\right) \leq \frac{1}{2} \log \frac{D_{1}}{D_{2}}
\end{aligned}
$$

by Lemma 1 and Theorem 1 since zero rate loss at the second stage implies $I\left(X ; Y_{2}, Y_{1}\right)=R\left(D_{2}\right)$.

The shaded region of Fig. 3(a) shows the possible values of $L_{1}$ as a function of $D_{2} / D_{1}$. The maximal value occurs when $D_{1}=5 D_{2}$, giving $L_{1} \leq(1 / 2) \log 5<1.1610$.

By applying the same basic strategy used in the proof of Theorem 3, we improve the bound given by [13, Theorem 5] from $L_{1}=0$ and $L_{2} \leq 1$ to $L_{1}=0$ and $L_{2} \leq(1 / 2) \log (\sqrt{3}+1)$. Fig. 2(b) shows the graphical interpretation of this result.

Theorem 4: For any $\left(D_{1}, D_{2}\right)$ with $D_{2}<D_{1}$, there exists an achievable rate-distortion vector $\left(R_{1}, R_{2}, D_{1}, D_{2}\right)$ with $L_{1}=$ 0 and $L_{2} \leq(1 / 2) \log (\sqrt{3}+1)$.

Proof: The proof of [13, Theorem 5] actually shows that

$$
L_{2} \leq \frac{1}{2} \log \left(1+\frac{D_{2}}{D_{1}}\right)+\frac{1}{2}
$$

for a code with $Y_{1}=U_{1}$ and $Y_{2}=U_{2}$, where $U_{1}$ and $U_{2}$ are the random variables that achieve $R\left(D_{1}\right)$ and $R\left(D_{2}\right)$, respectively, and $U_{1} \rightarrow X \rightarrow U_{2}$.

We here design a different code and derive a bound for the new code that decreases in $D_{2} / D_{1}$. We then put the increasing and decreasing bounds together by choosing, for each value of $D_{2} / D_{1}$, the smaller of the two bounds. Setting $U_{1}$ and $U_{2}$ to be the random variables that achieve $R\left(D_{1}\right)$ and $R\left(D_{2}\right)$, respectively, $\alpha=D_{2} / D_{1}$, and $N_{2} \sim \mathcal{N}\left(0, D_{2}-\alpha^{2} D_{1}\right)$, with $N_{2} \Perp\left(X, U_{1}\right)$ as in the proof of Lemma 1 and defining $Y_{2}=(1-\alpha) X+\alpha U_{1}+N_{2}$ and $Y_{1}=U_{1}$ gives $L_{1}=0$ and

$$
\begin{aligned}
L_{2} & =I\left(X ; Y_{2}, Y_{1}\right)-R\left(D_{2}\right)=I\left(X ; Y_{2}, U_{1}\right)-R\left(D_{2}\right) \\
& =I\left(X ; Y_{2} \mid U_{1}\right)+I\left(X ; U_{1}\right)-R\left(D_{2}\right) \\
& \leq I\left(X ; Y_{2} \mid U_{1}\right)
\end{aligned}
$$

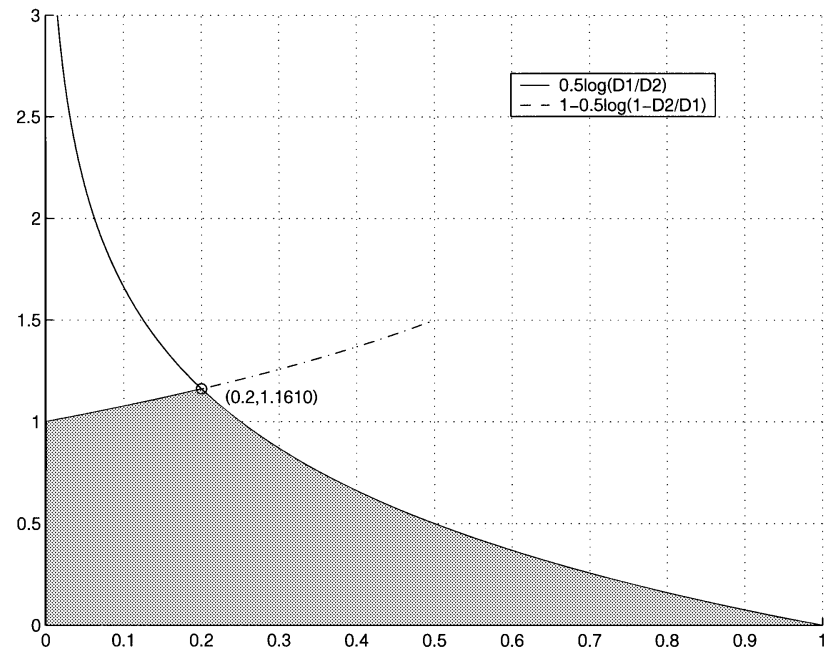

(a)

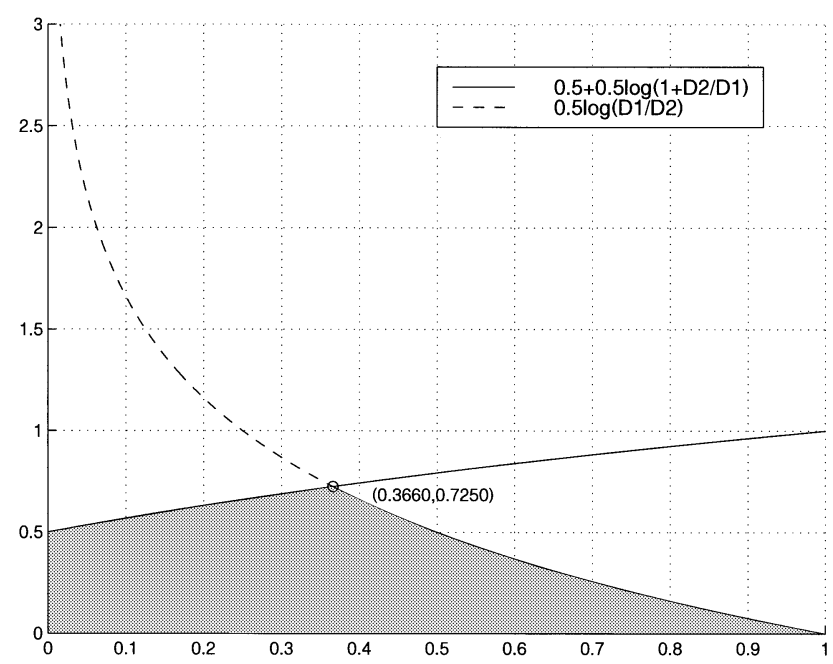

(b)

Fig. 3. Possible values of (a) $L_{1}$ in Theorem 3 and (b) $L_{2}$ in Theorem 4 .

$$
\leq \frac{1}{2} \log \frac{D_{1}}{D_{2}}
$$

Here (12) follows since $I\left(X ; U_{1}\right)=R\left(D_{1}\right), D_{2}<D_{1}$, and the rate-distortion function $R(D)$ is a nonincreasing function of $D$, and (13) follows from steps (32)-(35) of the proof of Lemma 1.

Thus, for any $D_{2} / D_{1}<1$, we have

$$
L_{2} \leq\left\{\begin{array}{l}
\frac{1}{2} \log \left(1+\frac{D_{2}}{D_{1}}\right)+\frac{1}{2} \\
\frac{1}{2} \log \frac{D_{1}}{D_{2}} .
\end{array}\right.
$$

The first bound dominates when $D_{1} / D_{2} \geq \sqrt{3}+1$; the second dominates when $1<D_{1} / D_{2}<\sqrt{3}+1$ (see Fig. 3(b)). Together these bounds give $L_{2} \leq(1 / 2) \log (\sqrt{3}+1)<0.7250$.

We can obtain a looser bound which can be generalized to any difference distortion measure by using $Y_{2}=X+N$, where $E d(N, 0) \leq D_{2}$ and $N \Perp\left(X, U_{1}\right)$; in this case, we can bound $L_{2}$ by $(1 / 2) \log \left(1+D_{1} / D_{2}\right)$, which leads to the constant bound $(1 / 2) \log 3<0.7925$.

We next refine the shape of this bound using a technique employed in [13, Theorem 6]. 
Theorem 5: For any $\left(D_{1}, D_{2}\right)$ with $D_{2}<D_{1}$, there exists an achievable rate-distortion vector $\left(R_{1}, R_{2}, D_{1}, D_{2}\right)$ with $L_{1}=0$ and

$$
L_{2} \leq \frac{1}{2} \log \left[\left(\sqrt{\frac{6 \sigma^{2}-5 D_{1}}{2 \sigma^{2}-D_{1}}}+1\right)\left(1-\frac{D_{1}}{2 \sigma^{2}}\right)\right] .
$$

Proof: From Theorem 4, $L_{2} \leq(1 / 2) \log \left(D_{1} / D_{2}\right)$. For any fixed $0<D_{1} \leq \sigma^{2}$, this bound is a decreasing function of $D_{2} \in\left(0, D_{1}\right)$. We next design another code and derive its corresponding bound, which is an increasing function of $D_{2}$. Let $U_{1} \rightarrow X \rightarrow U_{2}, \beta_{1}=1-D_{1} / \sigma^{2}, N_{1}^{\prime} \sim \mathcal{N}\left(0, \beta_{1} D_{1}\right)$, and $N_{1}^{\prime} \Perp\left(X, U_{1}, U_{2}\right)$. Then, setting $Y_{2}=U_{2}$ and $Y_{1}=U_{1}$ and using an approach from the proof of [13, Theorem 5] gives

$$
\begin{aligned}
L_{2} & =I\left(X ; U_{1} \mid U_{2}\right) \leq I\left(X ; U_{1}, \beta_{1} X+N_{1}^{\prime} \mid U_{2}\right) \\
& =I\left(X ; \beta_{1} X+N_{1}^{\prime} \mid U_{2}\right)+I\left(X ; U_{1} \mid U_{2}, \beta_{1} X+N_{1}^{\prime}\right) \\
& \leq I\left(X-U_{2} ; \beta_{1}\left(X-U_{2}\right)+N_{1}^{\prime}\right)+I\left(X ; \beta_{1} X+N_{1}^{\prime} \mid U_{1}\right) \\
& \leq \frac{1}{2} \log \left(1+\frac{D_{2}}{D_{1}}-\frac{D_{2}}{\sigma^{2}}\right)+\frac{1}{2} \log \left(2-\frac{D_{1}}{\sigma^{2}}\right) .
\end{aligned}
$$

The second bound dominates when $D_{2} \in(0, \Gamma]$, where

$$
\Gamma=\frac{D_{1} \sigma^{2} \sqrt{6 \sigma^{2}-5 D_{1}}-D_{1} \sigma^{2} \sqrt{2 \sigma^{2}-D_{1}}}{2\left(\sigma^{2}-D_{1}\right) \sqrt{2 \sigma^{2}-D_{1}}}
$$

while the first dominates for the remainder of the $\left(0, D_{1}\right)$ region. The maximal value of the combined bound is achieved at $D_{2}=\Gamma$, giving the desired result.

The bound described in Theorem 5 is tight when $D_{1}=\sigma^{2}$, where $L_{2}=0$. This bound can also be written as

$$
L_{2} \leq \frac{1}{2} \log \left[\left(\sqrt{5-\frac{4 \sigma^{2}}{2 \sigma^{2}-D_{1}}}+1\right)\left(1-\frac{D_{1}}{2 \sigma^{2}}\right)\right],
$$

which is a decreasing function of $D_{1}$ for a fixed value of $\sigma^{2}$. This bound achieves its maximum when $D_{1}=0$, giving $L_{2} \leq$ $(1 / 2) \log (\sqrt{3}+1)<0.7250$, which is consistent with Theorem 4 . The bound from Theorem 5 is less than the bound from Theorem 4 when $D_{1} \neq 0$. Fig. 4 shows the bound from Theorem 5. The shaded region shows the possible values of $L_{2}$ as a function of $D_{1} / \sigma^{2}$.

Lemma 2 shows the convexity of the rate loss. This result proves useful in Theorem 6, where we address the case where the rate losses at both resolutions are equal.

Lemma 2: For any $D_{2}<D_{1}$, if rate-distortion vectors $\left(R_{10}, R_{20}, D_{1}, D_{2}\right)$ and $\left(R_{11}, R_{21}, D_{1}, D_{2}\right)$ are 2RSCachievable on source $X$, then for any $0 \leq \alpha \leq 1$, there exists an achievable rate-distortion vector $\left(R_{1 \alpha}, R_{2 \alpha}, D_{1}, D_{2}\right)$ for the same source with $L_{1 \alpha}=\alpha L_{11}+(1-\alpha) L_{10}$ and $L_{2 \alpha}=\alpha L_{21}+(1-\alpha) L_{20}$. Here $L_{1 \beta}=R_{1 \beta}-R\left(D_{1}\right)$ and $L_{2 \beta}=R_{2 \beta}-R\left(D_{2}\right)$, for all $\beta \in\{0, \alpha, 1\}$.

Using the convexity of the rate loss proved in Lemma 2 with the bounds on $L_{1}$ and $L_{2}$ from Theorems 3 and 4 gives new bounds for the case where $L_{1}=L_{2}$. In particular, since for

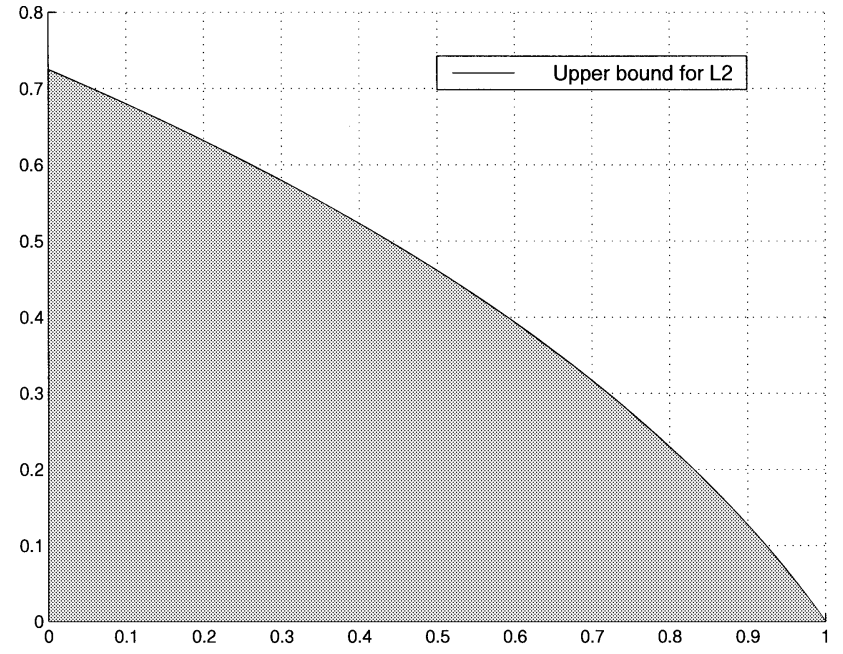

Fig. 4. Possible values of $L_{2}$ in Theorem 5 .

any $D_{2}<D_{1}$, the rate losses $L_{10} \leq(1 / 2) \log 5$ and $L_{20}=0$ and the rate losses $L_{11}=0$ and $L_{21} \leq(1 / 2) \log (\sqrt{3}+1)$ are both achievable by multiresolution coding with distortions $\left(D_{1}, D_{2}\right)$, the rate losses $L_{1 \alpha}=(1-\alpha) L_{10}$ and $L_{2 \alpha}=\alpha L_{21}$ are also achievable at these distortions. Setting

$$
\alpha=\frac{L_{10}}{L_{10}+L_{21}}
$$

proves the achievability of

$$
\begin{aligned}
L_{1 \alpha} & =L_{2 \alpha}=\frac{L_{10} L_{21}}{L_{10}+L_{21}}=\frac{1}{1 / L_{10}+1 / L_{21}} \\
& \leq \frac{1}{1 /\left(\frac{1}{2} \log 5\right)+1 /\left(\frac{1}{2} \log (\sqrt{3}+1)\right)}<0.4463
\end{aligned}
$$

with distortions $\left(D_{1}, D_{2}\right)$. This result slightly tightens the bound of [13, Theorem 3], which proves the achievability of $L_{1} \leq 1 / 2$ and $L_{2} \leq 1 / 2$ for distortions $\left(D_{1}, D_{2}\right)$.

Theorem 6 improves the bound further. The graphical interpretation is shown in Fig. 2(c).

Theorem 6: For any $D_{2}<D_{1}$, the rate losses $L_{1}=L_{2}<$ 0.3802 are achievable.

Proof: As shown in Theorem 3, the rate losses $L_{10}$ and $L_{20}$ are achievable, where

$$
L_{20}=0
$$

and

$$
L_{10} \leq \begin{cases}A_{1}=\frac{1}{2} \log \frac{4 D_{1}}{D_{1}-D_{2}}, & \text { if } D_{1} \geq 5 D_{2} \\ A_{2}=\frac{1}{2} \log \frac{D_{1}}{D_{2}}, & \text { if } D_{1}<5 D_{2} .\end{cases}
$$

From Theorem 4, the rate losses $L_{11}$ and $L_{21}$ are achievable, where

$L_{11}=0$

$L_{21} \leq \begin{cases}B_{1}=\frac{1}{2} \log \left(2+\frac{2 D_{2}}{D_{1}}\right), & \text { if } D_{1} \geq(\sqrt{3}+1) D_{2} \\ B_{2}=\frac{1}{2} \log \frac{D_{1}}{D_{2}}, & \text { if } D_{1}<(\sqrt{3}+1) D_{2} .\end{cases}$ 


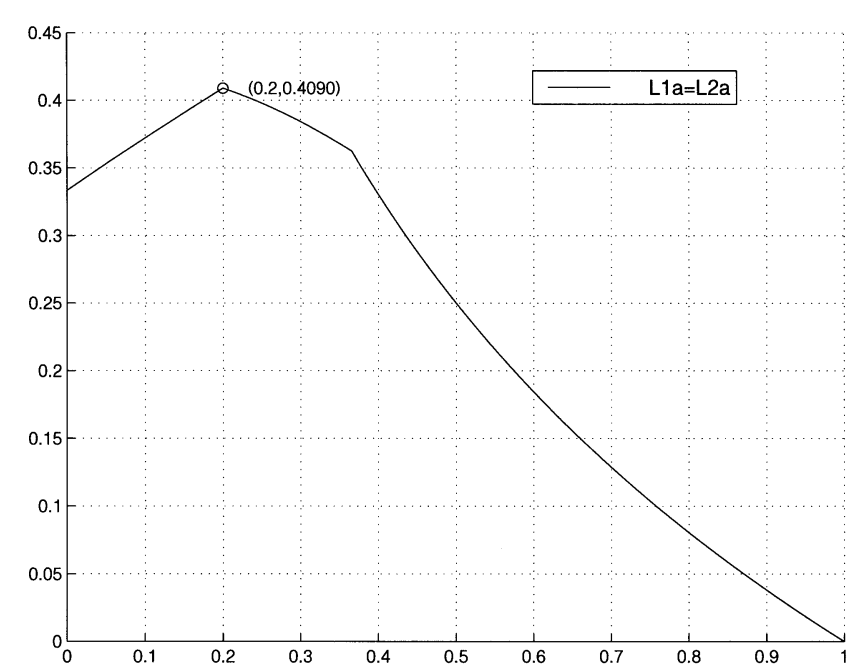

(a)

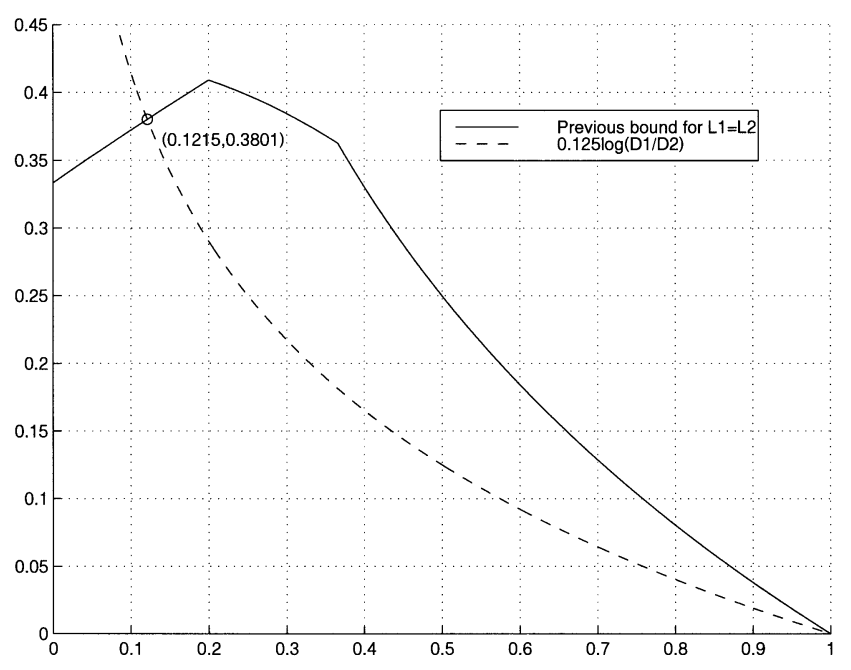

(b)

Fig. 5. Upper bounds for $L_{1 \alpha}=L_{2 \alpha}$ in Theorem 6 .

Thus, from Lemma 2, we can draw the conclusion that the rate losses $L_{1 \alpha}=(1-\alpha) L_{10}$ and $L_{2 \alpha}=\alpha L_{21}$ are achievable. Setting $\alpha=L_{10} /\left(L_{10}+L_{21}\right)$ gives

$$
L_{1 \alpha}=L_{2 \alpha} \leq \begin{cases}\frac{1}{1 / A_{1}+1 / B_{1}}, & \text { if } \sigma^{2} \geq D_{1} \geq 5 D_{2} \\ \frac{1}{1 / A_{2}+1 / B_{1}}, & \text { if }(\sqrt{3}+1) D_{2} \leq D_{1}<5 D_{2} \\ \frac{1}{1 / A_{2}+1 / B_{2}}, & \text { if } D_{2}<D_{1}<(\sqrt{3}+1) D_{2} .\end{cases}
$$

Fig. 5(a) shows the bound for $L_{1 \alpha}=L_{2 \alpha}$. The maximum occurs when $D_{2} / D_{1}=0.2$, giving

$$
L_{1 \alpha}=L_{2 \alpha} \leq \frac{1}{1 /\left(\frac{1}{2} \log 5\right)+1 /\left(\frac{1}{2} \log 2.4\right)}<0.4091
$$

Finally, we use the results of Theorems 3 and 4 in a different way to get the desired result. From (11), $L_{10} \leq R\left(D_{2}\right)-R\left(D_{1}\right)$ and $L_{20}=0$ are achievable. By (12) and (13), $L_{11}=0$ and

$$
L_{21} \leq(1 / 2) \log \left(D_{1} / D_{2}\right)+R\left(D_{1}\right)-R\left(D_{2}\right)
$$

are achievable. Thus, by convexity, we can achieve

$$
\begin{aligned}
L_{1 \alpha} & =L_{2 \alpha}=\frac{L_{10} L_{21}}{L_{10}+L_{21}} \\
& \leq \frac{\left[R\left(D_{2}\right)-R\left(D_{1}\right)\right]\left[\frac{1}{2} \log \frac{D_{1}}{D_{2}}+R\left(D_{1}\right)-R\left(D_{2}\right)\right]}{\frac{1}{2} \log \frac{D_{1}}{D_{2}}} \\
& \leq \frac{\left(R\left(D_{2}\right)-R\left(D_{1}\right)+\frac{1}{2} \log \frac{D_{1}}{D_{2}}+R\left(D_{1}\right)-R\left(D_{2}\right)\right)^{2}}{4\left(\frac{1}{2} \log \frac{D_{1}}{D_{2}}\right)} \\
& =\frac{1}{8} \log \frac{D_{1}}{D_{2}}
\end{aligned}
$$

where (14) follows since $4 a b \leq(a+b)^{2}$ for $a, b \geq 0$. Fig. 5(b) combines this bound with the bound of Fig. 5(a). The new maximum is achieved when $D_{2} / D_{1} \approx 0.1215$ and gives $L_{1 \alpha}=$ $L_{2 \alpha}<0.3802$, thus, $L_{1 \alpha}=L_{2 \alpha}<0.3802$ is achievable.

For any $M \geq 2$ and $0<D_{M}<\cdots<D_{2}<D_{1}$, [13, Corollary 1] shows that there exists an achievable rate-distortion vector $\left(R_{1}, \ldots R_{M}, D_{1}, \ldots, D_{M}\right)$ with $L_{i} \leq 1 / 2$, $i \in\{1, \ldots, M\}$. This solution suggests approximately identical priorities at all resolutions. We next consider the case where we minimize the rate loss at the first resolution, then minimize the rate loss at the second resolution subject to the first rate loss and so on. This greedy approach, used in the design of tree-structured vector quantizers (TSVQs) [20], apparently maximizes the rate loss at the last resolution. The next theorem provides an upper bound for this scenario. This result can also be regarded as a generalization of Theorem 4 . We first introduce Lemma 3 and Lemma 4, which are useful for proving this theorem.

Lemma 3: Let $A, B, C$, and $D$ be random variables such that $A \rightarrow B \rightarrow C, A \rightarrow C \rightarrow D$, and $A \rightarrow(B, C) \rightarrow D$ form Markov chains. Then $I(A ; B \mid D) \geq I(A ; B \mid C)$.

Lemma 4: For any $D_{M}<\cdots<D_{3}<D_{2}<D_{1}$, let $U_{1}$ be a random variable achieving $R\left(D_{1}\right)$ and $U_{i}^{\prime}$ for $i \in\{2,3, \ldots, M\}$ be successively defined as

$$
U_{i}^{\prime}=\arg \min _{U: E d(X, U) \leq D_{i}} I\left(X ; U_{1}, U_{2}^{\prime}, \ldots, U_{i-1}^{\prime}, U\right) .
$$

Then

$$
I\left(X ; U_{1}, U_{2}^{\prime}, \ldots, U_{M}^{\prime} \mid X+N_{M}\right) \leq M / 2,
$$

where $N_{M} \sim \mathcal{N}\left(0, D_{M}\right)$ and $N_{M} \Perp\left(X, U_{1}, U_{2}^{\prime}, \ldots, U_{M}^{\prime}\right)$.

Theorem 7: For any $D_{M}<\cdots<D_{2}<D_{1}$, let $U_{1}$ be a random variable achieving $R\left(D_{1}\right)$, and define $U_{2}^{\prime}, \ldots, U_{M-1}^{\prime}$ as in (15), and $L_{i}=I\left(X ; U_{1}, U_{2}^{\prime}, \ldots, U_{i}^{\prime}\right)-R\left(D_{i}\right)$ for all $1<i \leq M$. Then the rate losses for a greedily designed MRSC with distortions $\left(D_{1}, \ldots, D_{M}\right)$ are bounded as $L_{1}=0$ and $L_{i} \leq i / 2$ for all $1<i \leq M$.

Proof: For each $i \in\{2, \ldots, M\}$, let $U_{i}$ be the random variable that achieves $R\left(D_{i}\right)$, and define $N_{i-1} \sim \mathcal{N}\left(0, D_{i-1}\right)$, where

$$
N_{i-1} \Perp\left(X, U_{1}, U_{2}^{\prime}, \ldots, U_{i-1}^{\prime}, U_{i}\right) .
$$


Further let

$$
\left(U_{1}, U_{2}^{\prime}, \ldots, U_{i-1}^{\prime}\right) \rightarrow X \rightarrow U_{i}
$$

i.e., define the joint distribution of $\left(X, U_{1}, U_{2}^{\prime}, \ldots, U_{i-1}^{\prime}, U_{i}\right)$ as $f_{X} Q_{U_{1}, U_{2}^{\prime}, \ldots, U_{i-1}^{\prime} \mid X} Q_{U_{i} \mid X}$.

The greedy approach uses $Y_{1}=U_{1}$ and $Y_{i}=U_{i}^{\prime}$ for all $i \in\{2,3, \ldots, i-1\}$. The rate loss of this code at resolution $i$ is

$$
\begin{aligned}
L_{i}= & I\left(X ; Y_{i}, \ldots, Y_{2}, Y_{1}\right)-I\left(X ; U_{i}\right) \\
= & I\left(X ; U_{i}^{\prime}, U_{i-1}^{\prime}, \ldots, U_{2}^{\prime}, U_{1}\right)-I\left(X ; U_{i}\right) \\
\leq & I\left(X ; U_{i}, U_{i-1}^{\prime}, \ldots, U_{2}^{\prime}, U_{1}\right)-I\left(X ; U_{i}\right) \\
= & I\left(X ; U_{i-1}^{\prime}, \ldots, U_{2}^{\prime}, U_{1} \mid U_{i}\right) \\
\leq & I\left(X ; U_{i-1}^{\prime}, \ldots, U_{2}^{\prime}, U_{1}, X+N_{i-1} \mid U_{i}\right) \\
= & I\left(X ; X+N_{i-1} \mid U_{i}\right) \\
& +I\left(X ; U_{i-1}^{\prime}, \ldots, U_{2}^{\prime}, U_{1} \mid U_{i}, X+N_{i-1}\right)
\end{aligned}
$$

where (16) follows from the definition of $U_{i}^{\prime}$. We bound the first term in (17) as

$$
I\left(X ; X+N_{i-1} \mid U_{i}\right) \leq \frac{1}{2} \log \left(1+\frac{D_{i}}{D_{i-1}}\right) \leq \frac{1}{2}
$$

where the first inequality follows from the approach of steps (32)-(35) from the Appendix, and the second inequality follows since $D_{i}<D_{i-1}$. We bound the second term in (17) as

$$
\begin{aligned}
I(X ; & \left.U_{i-1}^{\prime}, \ldots, U_{2}^{\prime}, U_{1} \mid U_{i}, X+N_{i-1}\right) \\
\leq & I\left(X ; U_{i-1}^{\prime}, \ldots, U_{2}^{\prime}, U_{1} \mid U_{i}, X+N_{i-1}\right) \\
& +I\left(U_{i} ; U_{i-1}^{\prime}, \ldots, U_{2}^{\prime}, U_{1} \mid X+N_{i-1}\right) \\
= & I\left(X, U_{i} ; U_{i-1}^{\prime}, \ldots, U_{2}^{\prime}, U_{1} \mid X+N_{i-1}\right) \\
= & I\left(X ; U_{i-1}^{\prime}, \ldots, U_{2}^{\prime}, U_{1} \mid X+N_{i-1}\right) \\
& \quad+I\left(U_{i} ; U_{i-1}^{\prime}, \ldots, U_{2}^{\prime}, U_{1} \mid X, X+N_{i-1}\right) \\
= & I\left(X ; U_{i-1}^{\prime}, \ldots, U_{2}^{\prime}, U_{1} \mid X+N_{i-1}\right) \\
\leq & \frac{i-1}{2}
\end{aligned}
$$

where (19) follows since $U_{i}$ and $\left(U_{1}, U_{2}^{\prime}, \ldots, U_{i-1}^{\prime}\right)$ are independent given $\left(X, X+N_{i-1}\right)$, and (20) follows from Lemma 4. Combining (17), (18), and (20) proves the theorem.

Theorem 7 gives a collection of rate loss bounds that increase with the increasing resolution. This is consistent with our intuition that performance degrades at higher resolutions for greedily designed codes and suggests that the performance penalty associated with using greedily grown TSVQs [20] rather than jointly optimized multiresolution vector quantizers [5], [6] may be large. Proving such a result would require a tight bound on the rate losses studied in Theorem 7. We next show how to obtain a tighter bound on the rate losses in all stages of this greedily designed code by refining some of the previous arguments. We define $U_{i}^{\prime \prime}=(1-\alpha) X+\alpha U_{i-1}^{\prime}+N_{i}^{\prime}$, where $\alpha=D_{i} / D_{i-1}$, $N_{i}^{\prime} \sim \mathcal{N}\left(0, D_{i}-\alpha^{2} D_{i-1}\right)$, and $N_{i}^{\prime} \Perp\left(X, U_{1}, U_{2}^{\prime}, \ldots, U_{i}^{\prime}\right)$.
Since $U_{i}^{\prime \prime}$ and $\left(U_{i-2}^{\prime}, \ldots, U_{2}^{\prime}, U_{1}\right)$ are independent given $\left(X, U_{i-1}^{\prime}\right)$

$$
\begin{aligned}
L_{i}= & I\left(X ; U_{i}^{\prime}, U_{i-1}^{\prime}, \ldots, U_{2}^{\prime}, U_{1}\right)-R\left(D_{i}\right) \\
\leq & I\left(X ; U_{i}^{\prime \prime}, U_{i-1}^{\prime}, \ldots, U_{2}^{\prime}, U_{1}\right)-R\left(D_{i}\right) \\
= & I\left(X ; U_{i}^{\prime \prime} \mid U_{i-1}^{\prime}, \ldots, U_{2}^{\prime}, U_{1}\right)+L_{i-1} \\
& +\left[R\left(D_{i-1}\right)-R\left(D_{i}\right)\right] \\
\leq & I\left(X ; U_{i}^{\prime \prime} \mid U_{i-1}^{\prime}, \ldots, U_{2}^{\prime}, U_{1}\right)+L_{i-1} \\
\leq & I\left(X-U_{i-1}^{\prime} ;(1-\alpha)\left(X-U_{i-1}^{\prime}\right)\right. \\
& \left.\quad+N_{i}^{\prime} \mid U_{i-1}^{\prime}, \ldots, U_{2}^{\prime}, U_{1}\right)+L_{i-1} \\
\leq & \frac{1}{2} \log \frac{D_{i-1}}{D_{i}}+L_{i-1} \\
\leq & \frac{1}{2} \log \frac{D_{i-1}}{D_{i}}+\frac{i-1}{2}
\end{aligned}
$$

where (21) follows from the approach of steps (32)-(35) from the Appendix. Note that (18) also implies that

$$
L_{i} \leq(1 / 2) \log \left(1+D_{i} / D_{i-1}\right)+(i-1) / 2 .
$$

Together, these bounds give

$$
L_{i} \leq(i-1) / 2+(1 / 2) \log ((\sqrt{5}+1) / 2)<i / 2-0.1528
$$

We also find from (21) that $L_{i} \leq(1 / 2) \log \left(D_{1} / D_{i}\right)$. Unfortunately, it may be difficult to extend these new bounds to distortion measures other than squared error.

We can also generalize Theorem 7 in a different way. The following theorem suggests that the penalty of the greedy approach is in some sense constrained to the resolutions in which it is applied.

Theorem 8: For any $D_{M}<\cdots<D_{2}<D_{1}$ and any $K \in$ $\{2, \ldots, M\}$, let $U_{1}$ be a random variable achieving $R\left(D_{1}\right)$ and define $U_{2}^{\prime}, \ldots, U_{K-1}^{\prime}$ as in (15). Set $Y_{1}=U_{1}$ and $Y_{i}=U_{i}^{\prime}$ for all $1<i<K$, but constrain $Y_{i}$ for $K \leq i \leq M$ only to guarantee distortion $D_{i}$. Then we can achieve rate losses bounded as $L_{1}=0, L_{i} \leq i / 2$ for all $1<i<K$, and $L_{i} \leq K / 2$ for all $K \leq i \leq M$.

Proof: Let $U_{i}$ be a random variable that achieves $R\left(D_{i}\right)$ for any $1 \leq i \leq M, N_{i} \sim \mathcal{N}\left(0, D_{i}-D_{i+1}\right)$ for $K-1 \leq$ $i<M, N_{M} \sim \mathcal{N}\left(0, D_{M}\right), N_{i} \Perp N_{j}$ for any $j \neq i$, and $N_{i} \Perp\left(X, U_{1}, U_{2}^{\prime}, \ldots, U_{K-1}^{\prime}\right)$. Further, define $Y_{1}=U_{1}, Y_{i}=$ $U_{i}^{\prime}$ for any $1<i<K$ and $Y_{i}=X+\sum_{j=i}^{M} N_{j}$ for $K \leq i \leq M$.

Then $L_{1}=0, L_{i} \leq i / 2$ for all $1<i<K$ by Theorem 7 , and for any $K \leq i \leq M$

$$
\begin{aligned}
& L_{i}= I\left(X ; Y_{1}, \ldots, Y_{i}\right)-R\left(D_{i}\right) \\
&=I\left(X ; U_{1}, U_{2}^{\prime}, \ldots, U_{K-1}^{\prime}, X\right. \\
&\left.\quad+\sum_{j=K}^{M} N_{j}, \ldots, X+\sum_{j=i}^{M} N_{j}\right)-I\left(X ; U_{i}\right) \\
&=I\left(X ; U_{1}, U_{2}^{\prime}, \ldots, U_{K-1}^{\prime}, X+\sum_{j=i}^{M} N_{j}\right)-I\left(X ; U_{i}\right)
\end{aligned}
$$




$$
\begin{aligned}
= & I\left(X ; U_{1}, U_{2}^{\prime}, \ldots, U_{K-1}^{\prime} \mid X+\sum_{j=i}^{M} N_{j}\right) \\
& +I\left(X ; X+\sum_{j=i}^{M} N_{j}\right)-I\left(X ; U_{i}\right) \\
\leq & I\left(X ; U_{1}, U_{2}^{\prime}, \ldots, U_{K-1}^{\prime} \mid X+\sum_{j=i}^{M} N_{j}\right)+\frac{1}{2} \\
\leq & I\left(X ; U_{1}, U_{2}^{\prime}, \ldots, U_{K-1}^{\prime} \mid X+\sum_{j=K-1}^{M} N_{j}\right)+\frac{1}{2} \\
\leq & \frac{K}{2}
\end{aligned}
$$

where (22) follows since

$$
X \rightarrow X+\sum_{j=i}^{M} N_{j} \rightarrow\left(X+\sum_{j=K}^{M} N_{j}, \ldots, X+\sum_{j=i+1}^{M} N_{j}\right)
$$

forms a Markov chain, (23) follows since

$$
\sum_{j=i}^{M} N_{j} \sim \mathcal{N}\left(0, D_{i}\right)
$$

(24) follows from Lemma 3, and (25) follows from Lemma 4.

We next obtain a bound for the scenario where we first set $L_{M}$ to 0 , then minimize $L_{M-1}$ subject to $L_{M}$, and so on. This result, which can be viewed as a generalization of Theorem 3 , mirrors the approach of code designs like [21].

Theorem 9: For any $D_{M}<\cdots<D_{2}<D_{1}$ and any $K \in\{1, \ldots, M-1\}$, let $U_{M}$ be a random variable achieving $R\left(D_{M}\right)$ and for all $i \in\{M-1, M-2, \ldots, K\}$ let $V_{i}^{\prime}$ be sequentially defined as

$$
V_{i}^{\prime}=\arg _{V: E d(X, V) \leq D_{i}, X \rightarrow U_{M} \rightarrow V_{M-1}^{\prime} \cdots V_{i+1}^{\prime} \rightarrow V} I(X ; V) .
$$

Set $Y_{i}=V_{i}^{\prime}$ for all $K<i<M$ and constrain $Y_{i}$ for $1 \leq i \leq K$ only to guarantee distortion $D_{i}$. Then the rate losses

$$
\begin{array}{ll}
L_{i} \leq((M-i) / 2) \log 5, & \text { for all } K<i<M \\
L_{i} \leq((M-K) / 2) \log 5, & \text { for all } 1 \leq i \leq K
\end{array}
$$

are achievable.

Proof: For any $1 \leq i \leq M$, let $U_{i}$ be a random variable that achieves $R\left(D_{i}\right)$ and $N_{i} \sim \mathcal{N}\left(0, D_{i}-D_{i+1}\right)$, where $N_{i} \Perp\left(X, U_{M}, V_{M-1}^{\prime}, \ldots, V_{K+1}^{\prime}\right)$ and $N_{i} \Perp N_{j}$ for any $j \neq i$. Further, define $Y_{M}=U_{M}, Y_{i}=V_{i}^{\prime}$ for any $K<i<M$, and $Y_{i}=V_{K+1}^{\prime}+\sum_{j=i}^{K} N_{j}$ for $1 \leq i \leq K$. Since $X \rightarrow$ $Y_{M} \rightarrow \cdots \rightarrow Y_{1}, R_{i}=I\left(X ; Y_{1}, \ldots, Y_{i}\right)=I\left(X ; Y_{i}\right)$ for all $1 \leq i \leq M$. Thus, $L_{M}=0, L_{i}=I\left(X ; V_{i}^{\prime}\right)-R\left(D_{i}\right)$ for all $M>i>K$, and $L_{i}=I\left(X ; V_{K+1}^{\prime}+\sum_{j=i}^{K} N_{j}\right)-R\left(D_{i}\right)$ for any $1 \leq i \leq K$.

For all $K+1<i<M$

$L_{i-1}=I\left(X ; V_{i-1}^{\prime}\right)-R\left(D_{i-1}\right) \leq I\left(X ; V_{i}^{\prime}+N_{i-1}\right)-R\left(D_{i-1}\right)$.
Let $N_{i}^{\prime} \sim \mathcal{N}\left(0, D_{i}\right)$ be independent of all other random variables; then from the proof of Theorem 3

$$
\begin{aligned}
L_{i-1} \leq & I\left(X ; V_{i}^{\prime}+N_{i-1}\right)-I\left(X ; U_{i-1}\right) \\
= & {\left[I\left(X ; V_{i}^{\prime}+N_{i-1}\right)-I\left(X ; V_{i}^{\prime}\right)\right] } \\
& +\left[I\left(X ; X+N_{i}^{\prime}\right)-I\left(X ; X+N_{i}^{\prime}+N_{i-1}\right)\right] \\
& +\left[I\left(X ; X+N_{i}^{\prime}+N_{i-1}\right)-I\left(X ; U_{i-1}\right)\right] \\
& +\left[I\left(X ; V_{i}^{\prime}\right)-I\left(X ; X+N_{i}^{\prime}\right)\right] \\
\leq & 1+\frac{1}{2} \log \frac{D_{i-1}}{D_{i-1}-D_{i}}+I\left(X ; V_{i}^{\prime}\right)-I\left(X ; X+N_{i}^{\prime}\right) \\
\leq & 1+\frac{1}{2} \log \frac{D_{i-1}}{D_{i-1}-D_{i}}+I\left(X ; V_{i}^{\prime}\right)-R\left(D_{i}\right) \\
= & 1+\frac{1}{2} \log \frac{D_{i-1}}{D_{i-1}-D_{i}}+L_{i} .
\end{aligned}
$$

On the other hand, since $X \rightarrow V_{i}^{\prime} \rightarrow V_{i-1}^{\prime}$

$$
R_{i-1}=I\left(X ; V_{i-1}^{\prime}\right) \leq I\left(X ; V_{i}^{\prime}\right) .
$$

Therefore,

$$
\begin{aligned}
L_{i-1} & \leq I\left(X ; V_{i}^{\prime}\right)-R\left(D_{i-1}\right) \\
& =I\left(X ; V_{i}^{\prime}\right)-R\left(D_{i}\right)+R\left(D_{i}\right)-R\left(D_{i-1}\right) \\
& \leq L_{i}+\frac{1}{2} \log \frac{D_{i-1}}{D_{i}} .
\end{aligned}
$$

Together, these bounds imply that $L_{i-1} \leq L_{i}+(1 / 2) \log 5$, giving $L_{i} \leq((M-i) / 2) \log 5$ for all $K<i<M$ by induction. Similarly, $L_{i} \leq((M-K) / 2) \log 5$ for all $1 \leq i \leq K$.

Finally, we turn our attention briefly to A2RSCs. Before bounding the rate losses, we first demonstrate another property of the rate-distortion function.

Lemma 5: Suppose $R(D)$ is a rate-distortion function and $D_{0} \leq \min \left\{D_{1}, D_{2}\right\}$, then

$$
R\left(D_{1}\right)+R\left(D_{2}\right) \leq R\left(D_{0}\right), \quad \text { if } D_{1}+D_{2}-D_{0} \geq \sigma^{2} \text {. }
$$

Theorem 10: For any $D_{2}<D_{1}$, there exists an A2RSCachievable vector $\left(R_{1}, R_{2}, D_{1}, D_{2}\right)$ with $L_{1} \leq 1 / 2$ and $L_{2} \leq$ $(1 / 2) \log \Lambda$, where

$$
\begin{aligned}
\Lambda=\min \left\{1+\frac{D_{1}}{D_{2}}-\frac{D_{1}}{\sigma^{2}}, \frac{\sigma^{2}\left(\sigma^{2}-D_{1}+D_{2}\right)+\left(D_{1}-D_{2}\right) D_{2}}{\sigma^{2} D_{2}},\right. \\
\\
\left.\frac{D_{1}}{D_{2}}\left(2-\frac{D_{1}}{\sigma^{2}}\right)\left(1+\frac{D_{1}-D_{2}}{\sigma^{2}}\right)\left(1-\frac{D_{1}-D_{2}}{\sigma^{2}}\right)\right\} .
\end{aligned}
$$

Proof: Assume without loss of generality that $E(X)=0$. Let $U_{1}, U_{2}$, and $U_{0}^{\prime}$ be the random variables that achieve $R\left(D_{1}\right)$, $R\left(D_{2}\right)$, and $R\left(\sigma^{2}-D_{1}+D_{2}\right)$, respectively. Set $\beta_{i}=1-D_{i} / \sigma^{2}$, $i \in\{1,2\}$

$$
\begin{aligned}
N_{1} & \sim \mathcal{N}\left(0, \beta_{1} D_{1}-\beta_{1}^{2} D_{2} / \beta_{2}\right) \\
N_{2}^{\prime} & \sim \mathcal{N}\left(0, \beta_{2} D_{2}\right)
\end{aligned}
$$

and $N_{1} \Perp N_{2}^{\prime}$. Further, let

$$
\left(N_{1}, N_{2}^{\prime}\right) \Perp\left(X, U_{0}, U_{1}, U_{2}\right) .
$$

We define $Y_{2}=\beta_{2} X+N_{2}^{\prime}$ and

$$
Y_{1}=\beta_{1} Y_{2} / \beta_{2}+N_{1}=\beta_{1} X+\beta_{1} N_{2}^{\prime} / \beta_{2}+N_{1} .
$$


Then, by Theorem 2

$$
\begin{aligned}
\left(I\left(X ; Y_{1}\right), \max \left\{I\left(X ; Y_{1}\right)+I(X ; \Delta Y),\right.\right. \\
\left.\left.I\left(X ; Y_{1}, \Delta Y\right)+I\left(Y_{1} ; \Delta Y\right)\right\}, D_{1}, D_{2}\right)
\end{aligned}
$$

is achievable, where

$$
\Delta Y=Y_{2}-Y_{1}=\left(\beta_{2}-\beta_{1}\right) X+\left(\beta_{2}-\beta_{1}\right) N_{2}^{\prime} / \beta_{2}-N_{1} .
$$

Since

$$
\begin{aligned}
& I\left(X ; Y_{1}, \Delta Y\right)+I\left(Y_{1} ; \Delta Y\right)-I\left(X ; Y_{1}\right) \\
& \quad=I\left(X ; \Delta Y \mid Y_{1}\right)+I\left(Y_{1} ; \Delta Y\right) \\
& \quad=I\left(\Delta Y ; X, Y_{1}\right) \geq I(X ; \Delta Y)
\end{aligned}
$$

then

$$
\left(I\left(X ; Y_{1}\right), I\left(X ; Y_{1}, \Delta Y\right)+I\left(Y_{1} ; \Delta Y\right), D_{1}, D_{2}\right)
$$

is achievable. Using the argument from steps (31)-(35) in the Appendix and the fact that $E d(X, \Delta Y)=\sigma^{2}-D_{1}+D_{2}$

$$
\begin{aligned}
L_{1} & =I\left(X ; Y_{1}\right)-I\left(X ; U_{1}\right) \\
& \leq \frac{1}{2} \log \left(2-\frac{D_{1}}{\sigma^{2}}\right) \leq \frac{1}{2}, \\
I(X ; \Delta Y)-I\left(X ; U_{0}^{\prime}\right) & \leq \frac{1}{2} \log \left(2-\frac{\sigma^{2}-D_{1}+D_{2}}{\sigma^{2}}\right) \\
& =\frac{1}{2} \log \left(1+\frac{D_{1}-D_{2}}{\sigma^{2}}\right) .
\end{aligned}
$$

The rate loss at the second stage is

$$
\begin{aligned}
L_{2} & =I\left(X ; Y_{1}, \Delta Y\right)+I\left(Y_{1} ; \Delta Y\right)-R\left(D_{2}\right) \\
& =I\left(X ; Y_{1}\right)+I(X ; \Delta Y)+I\left(Y_{1} ; \Delta Y \mid X\right)-I\left(X ; U_{2}\right)
\end{aligned}
$$

where

$$
\begin{aligned}
I\left(Y_{1} ; \Delta Y \mid X\right) & =I\left(\frac{\beta_{1}}{\beta_{2}} N_{2}^{\prime}+N_{1} ; \frac{\beta_{2}-\beta_{1}}{\beta_{2}} N_{2}^{\prime}-N_{1}\right) \\
& =\frac{1}{2} \log \frac{D_{1}}{D_{2}}\left(1-\frac{D_{1}-D_{2}}{\sigma^{2}}\right) .
\end{aligned}
$$

Then $L_{2}$ can be bounded as

$$
\begin{aligned}
L_{2}= & {\left[I\left(X ; Y_{1}\right)-I\left(X ; U_{2}\right)\right]+I\left(Y_{1} ; \Delta Y \mid X\right)+I(X ; \Delta Y) } \\
\leq & I\left(X ; Y_{1} \mid U_{2}\right)+I\left(Y_{1} ; \Delta Y \mid X\right) \\
& +I\left(X ;\left(\beta_{2}-\beta_{1}\right) X+\left(\beta_{2}-\beta_{1}\right) N_{2}^{\prime} / \beta_{2}-N_{1}\right) \\
\leq & \frac{1}{2} \log \left(1+\frac{D_{2}}{D_{1}}-\frac{D_{2}}{\sigma^{2}}\right)+\frac{1}{2} \log \frac{D_{1}}{D_{2}}\left(1-\frac{D_{1}-D_{2}}{\sigma^{2}}\right) \\
& +\frac{1}{2} \log \frac{1}{1-\left(D_{1}-D_{2}\right) / \sigma^{2}} \\
= & \frac{1}{2} \log \left(1+\frac{D_{1}}{D_{2}}-\frac{D_{1}}{\sigma^{2}}\right) .
\end{aligned}
$$

On the other hand

$$
\begin{aligned}
L_{2}= & I\left(X ; Y_{1}\right)+\left[I(X ; \Delta Y)-I\left(X ; U_{2}\right)\right]+I\left(Y_{1} ; \Delta Y \mid X\right) \\
\leq & I\left(X ; \beta_{1} X+\beta_{1} N_{2}^{\prime} / \beta_{2}+N_{1}\right)+I\left(X ; \Delta Y \mid U_{2}\right) \\
& +I\left(Y_{1} ; \Delta Y \mid X\right) \\
\leq & \frac{1}{2} \log \frac{\sigma^{2}}{D_{1}}+\frac{1}{2} \log \left(1+\frac{\left(D_{1}-D_{2}\right) D_{2}}{\sigma^{2}\left(\sigma^{2}-D_{1}+D_{2}\right)}\right) \\
& +\frac{1}{2} \log \frac{D_{1}}{D_{2}}\left(1-\frac{D_{1}-D_{2}}{\sigma^{2}}\right) \\
= & \frac{1}{2} \log \frac{\sigma^{2}\left(\sigma^{2}-D_{1}+D_{2}\right)+\left(D_{1}-D_{2}\right) D_{2}}{\sigma^{2} D_{2}} .
\end{aligned}
$$

Combining Lemma 5, (26), and (27) with (28) gives the last bound.

We can combine the bounds given in (29) and (30) for fixed $D_{2}$. The first one dominates when $D_{1} \leq\left(\sigma^{2}+D_{2}\right) / 2$, while the second one dominates when $D_{1}>\left(\sigma^{2}+D_{2}\right) / 2$. The maximal value of the combined bound is achieved at $D_{1}=\left(\sigma^{2}+D_{2}\right) / 2$, giving $L_{2} \leq(1 / 2) \log \left(1+\left(\sigma^{4}-D_{2}^{2}\right) /\left(2 D_{2} \sigma^{2}\right)\right)$.

These bounds are good for the low-rate region, especially for large $D_{2}$. For example, if either $D_{2} / D_{1} \geq 1 / 3$ or $D_{2} / \sigma^{2} \geq$ $\sqrt{10}-3 \approx 0.1623$, we have $L_{1} \leq 1 / 2$ and $L_{2} \leq 1$. Although they depend on $D_{1}, D_{2}$, and $\sigma^{2}$, they may still be interesting since no tight characterization is known for the achievable region, and it is generally difficult to compute from the original result in [15].

Based on the proof of Theorem 10, we next obtain a new bound that depends on $h(X)$, the differential entropy of the source. The bound can be easily computed using only the variance and the differential entropy of the source. This bound is tight if $X$ is Gaussian.

Theorem 11: For any $D_{2}<D_{1}$, there exists an A2RSCachievable vector $\left(R_{1}, R_{2}, D_{1}, D_{2}\right)$ with

$$
\begin{aligned}
& L_{1} \leq \min \left\{\frac{1}{2}, \frac{1}{2} \log \left(2 \pi e \sigma^{2}\right)-h(X)\right\} \\
& L_{2} \leq \frac{1}{2} \log \left(2 \pi e \sigma^{2}\right)-h(X) .
\end{aligned}
$$

Proof: From the Shannon lower bound

$$
I\left(X ; U_{2}\right)=R\left(D_{2}\right) \geq h(X)-\frac{1}{2} \log \left(2 \pi e D_{2}\right) .
$$

From (28), there exists an A2RSC-achievable vector $\left(R_{1}, R_{2}, D_{1}, D_{2}\right)$ with $L_{1} \leq 1 / 2$ and

$$
\begin{aligned}
L_{2}= & I(X ; \Delta Y)+I\left(Y_{1} ; \Delta Y \mid X\right)+I\left(X ; Y_{1}\right)-I\left(X ; U_{2}\right) \\
\leq & \frac{1}{2} \log \frac{\sigma^{2}}{\sigma^{2}-D_{1}+D_{2}}+\frac{1}{2} \log \frac{D_{1}}{D_{2}}\left(1-\frac{D_{1}-D_{2}}{\sigma^{2}}\right) \\
& +\frac{1}{2} \log \frac{\sigma^{2}}{D_{1}}-h(X)+\frac{1}{2} \log \left(2 \pi e D_{2}\right) \\
= & \frac{1}{2} \log \left(2 \pi e \sigma^{2}\right)-h(X) .
\end{aligned}
$$

Similarly,

$$
L_{1} \leq I\left(X ; Y_{1}\right)-I\left(X ; U_{1}\right) \leq \frac{1}{2} \log \left(2 \pi e \sigma^{2}\right)-h(X) .
$$

An outer bound on the achievable region for A2RSCs also appears in [15]. It is easy to show the existence of a constant bound on the rate loss if the outer bound is tight. Set $Y_{2}=X+N_{2}, Y_{1}=X+N_{2}+N_{1}$, where $\left(N_{1}, N_{2}\right) \Perp X$, $N_{1} \Perp N_{2}, N_{2} \sim \mathcal{N}\left(0, D_{2}\right)$, and $N_{1} \sim \mathcal{N}\left(0, D_{1}-D_{2}\right)$. Then, $\Delta Y=-N_{1}$. Therefore, $L_{1}=I\left(X ; Y_{1}\right)-R\left(D_{1}\right) \leq 1 / 2$. Since $I(X ; \Delta Y)=0$,

$$
L_{2}=I\left(X ; Y_{1}, \Delta Y\right)-R\left(D_{2}\right)=I\left(X ; Y_{2}\right)-R\left(D_{2}\right) \leq 1 / 2 \text {. }
$$

\section{SUMMARY}

In this paper, we derive new rate loss bounds for MRSCs and A2RSCs, in some cases tightening existing results and in other cases treating cases where bounds did not exist previously. For 2RSCs, from the convexity of the rate loss and Theorems 3 and 4 , given any rate budget at one resolution, we can immediately 
upper-bound the rate at the other resolution of the optimal code. While most cases lead to small, constant bounds, we believe that these bounds are not tight in general. For example, Lastras and Berger show that $L_{1} \leq 1 / 2$ and $L_{2}=0$ are achievable as $D_{2} \rightarrow 0$, our bound is $L_{1} \rightarrow 1$ in this configuration.

Unlike most of Lastras and Berger's results, it may be difficult to extend most of our results to other distortion measure. For example, we construct $Y_{1}=U_{2}+N_{1}$ in the proof of Theorem 3 , but this construction cannot be extended to other distortion measure directly, e.g.,

$$
E\left[\left(X-\left(U_{2}+N_{1}\right)\right)^{4}\right] \neq E\left[\left(X-U_{2}\right)^{4}\right]+E\left[N_{1}^{4}\right] .
$$

However, the results of Theorems 7 and 8 and a looser version of Theorem 4 can be extended to any difference distortion measure.

Another interesting issue is the implication of the rate loss bounds for code design. The entropy-coded dithered-lattice quantizer (ECDQ) [22], [23] is a uniform quantizer followed by a noiseless variable-rate encoder. The input of the quantizer is the sum of the source and an independent, uniformly distributed random variable and the output is the quantizer reproduction minus the same random noise. If we apply the approach developed in [24] to MRSCs, we can in practice design a code with rate loss [25]

$$
\begin{aligned}
L_{i} & \leq \frac{1}{2} \sum_{j=1}^{i} \log \left(2 \pi e G_{K j}\right)+\frac{1}{2} \log \left(2-\frac{D_{i}}{\sigma^{2}}\right) \\
& \leq \frac{1}{2} \sum_{j=1}^{i} \log \left(2 \pi e G_{K j}\right)+0.5
\end{aligned}
$$

where $K$ is the codeword length and $G_{K j}$ is the normalized second moment of the $K$-dimensional lattice at the $j$ th resolution (e.g., see [22]). For example, if we use one-dimensional lattices, then $G_{1}=1 / 12$, and $L_{1}<0.7547$, $L_{2}<1.0094, \ldots, L_{i}<0.2547 i+0.5$. If we use optimal lattices and let $K \rightarrow \infty$, then $G_{K} \rightarrow 1 /(2 \pi e)$, and $L_{i} \leq 0.5$ at any resolution. Unfortunately, we have not found corresponding practical coding schemes for other scenarios.

\section{APPENDIX}

Proof of Lemma 1: Let $U_{1}$ be the random variable that achieves $R\left(D_{1}\right)$, and let $N_{2} \sim \mathcal{N}\left(0, D_{2}-\alpha^{2} D_{1}\right)$, with $N_{2} \Perp\left(X, U_{1}\right)$ and $\alpha=D_{2} / D_{1}$. Note that for any $D_{2}>0$

$$
D_{2}-\alpha^{2} D_{1}=D_{2}-\left(\frac{D_{2}}{D_{1}}\right)^{2} D_{1}=\frac{D_{2}}{D_{1}}\left(D_{1}-D_{2}\right)>0
$$

implies that we have a legitimate distribution. Next, let $Y_{2}=$ $(1-\alpha) X+\alpha U_{1}+N_{2}$. Notice that

$$
\begin{aligned}
E d\left(X, Y_{2}\right) & =E\left(\alpha\left(X-U_{1}\right)-N_{2}\right)^{2} \\
& =\alpha^{2} E\left(X-U_{1}\right)^{2}+D_{2}-\alpha^{2} D_{1} \\
& \leq \alpha^{2} D_{1}+D_{2}-\alpha^{2} D_{1}=D_{2} .
\end{aligned}
$$

Thus, $R\left(D_{2}\right) \leq I\left(X ; Y_{2}\right)$, which implies

$$
\begin{aligned}
& R\left(D_{2}\right)-R\left(D_{1}\right) \\
& \quad \leq I\left(X ; Y_{2}\right)-I\left(X ; U_{1}\right) \\
& \quad=I\left(X ; Y_{2} \mid U_{1}\right)-I\left(X ; U_{1} \mid Y_{2}\right) \\
& \quad \leq I\left(X ; Y_{2} \mid U_{1}\right) \\
& \quad=I\left(X ;(1-\alpha) X+\alpha U_{1}+N_{2} \mid U_{1}\right) \\
& \quad=I\left(X-U_{1} ;(1-\alpha)\left(X-U_{1}\right)+N_{2} \mid U_{1}\right)
\end{aligned}
$$

$$
\begin{aligned}
& \leq I\left(X-U_{1} ;(1-\alpha)\left(X-U_{1}\right)+N_{2}\right) \\
& \leq \sup I\left(W ;(1-\alpha) W+N_{2}\right) \\
& =\frac{1}{2} \log \frac{(1-\alpha)^{2} D_{1}+D_{2}-\alpha^{2} D_{1}}{D_{2}-\alpha^{2} D_{1}} \\
& =\frac{1}{2} \log \frac{D_{1}}{D_{2}}
\end{aligned}
$$

where (31) follows by applying the chain rule twice to $I\left(X ; Y_{2}, U_{1}\right)$, (32) follows since $h(A \mid B)=h(A-B \mid B)$, and (33) follows since $U_{1} \rightarrow X-U_{1} \rightarrow(1-\alpha)\left(X-U_{1}\right)+N_{2}$ forms a Markov chain by the independence assumptions. In (34), we take the supremum over all random variables $W \Perp N_{2}$ such that $E\left(W^{2}\right) \leq D_{1}$; the supremum is achieved by $W \sim \mathcal{N}\left(0, D_{1}\right)$, giving the desired result.

This bound is tight. The Gaussian source achieves this bound. If we set $Y_{2}=X+N$, we can show a looser bound which can be extended to any difference distortion measure, where $E d(N, 0)=D_{2}$ and $N \Perp\left(X, U_{1}\right)$. For example, the looser bound for the squared error distortion measure would be

$$
R\left(D_{2}\right)-R\left(D_{1}\right) \leq \frac{1}{2} \log \left(1+\frac{D_{1}}{D_{2}}\right)
$$

In this case, we can also notice that $f_{1}\left(D_{2}\right)=R\left(D_{2}\right)-R\left(D_{1}\right)$ is a convex function of $D_{2}$ for fixed $D_{1}$ and this function achieves 0 if $D_{2}=D_{1}$. Therefore, the combination of function $f_{2}\left(D_{2}\right)=(1 / 2) \log \left(1+D_{1} / D_{2}\right)$ and its tangent which passes the point $\left(D_{1}, 0\right)$ provides a better bound.

Proof of Lemma 2: Following [12, Lemma 2], if

$$
\left(R_{10}, R_{20}, D_{1}, D_{2}\right) \text { and }\left(R_{11}, R_{21}, D_{1}, D_{2}\right)
$$

are achievable, then $\left(R_{1 \alpha}, R_{2 \alpha}, D_{1}, D_{2}\right)$ is achievable because of the convexity of the achievable rate-distortion region of the multiresolution codes, where

$$
R_{1 \alpha}=\alpha R_{11}+(1-\alpha) R_{10}
$$

and

$$
R_{2 \alpha}=\alpha R_{21}+(1-\alpha) R_{20} \text {. }
$$

(While the proof in [12, Lemma 2] uses incremental rates, the result generalizes immediately to total rates. In particular, if the vectors $\left(R_{10}, \Delta R_{0}=R_{20}-R_{10}, D_{1}, D_{2}\right)$ and $\left(R_{11}, \Delta R_{1}=R_{21}-R_{11}, D_{1}, D_{2}\right)$ of incremental rates and total distortions are achievable, then $\left(R_{1 \alpha}, \Delta R_{\alpha}=\alpha \Delta R_{1}+(1-\alpha) \Delta R_{0}, D_{1}, D_{2}\right)$ is achievable by $[12$, Lemma 2$]$. The corresponding total rate is

$$
\begin{aligned}
R_{1 \alpha}+\Delta R_{\alpha} & =\alpha R_{11}+(1-\alpha) R_{10}+\alpha \Delta R_{1}+(1-\alpha) \Delta R_{0} \\
& =\alpha R_{21}+(1-\alpha) R_{20}=R_{2 \alpha}
\end{aligned}
$$

which gives the convexity result used above.)

The corresponding rate losses are

$$
\begin{aligned}
L_{1 \alpha} & =R_{1 \alpha}-R\left(D_{1}\right)=\alpha R_{11}+(1-\alpha) R_{10}-R\left(D_{1}\right) \\
& =\alpha L_{11}+(1-\alpha) L_{10} \\
L_{2 \alpha} & =R_{2 \alpha}-R\left(D_{2}\right)=\alpha R_{21}+(1-\alpha) R_{20}-R\left(D_{2}\right) \\
& =\alpha L_{21}+(1-\alpha) L_{20}
\end{aligned}
$$

giving the desired result. 
Proof of Lemma 3: By using the chain rule for mutual information twice, we get

$$
\begin{aligned}
I(A ; B, C \mid D) & =I(A ; B \mid D)+I(A ; C \mid B, D) \\
& =I(A ; C \mid D)+I(A ; B \mid C, D) .
\end{aligned}
$$

Now we can show $I(A ; D \mid B)=0$ since

$$
\begin{aligned}
I(A ; D \mid B) & \leq I(A ; D \mid B)+I(A ; C \mid B, D) \\
& =I(A ; C, D \mid B) \\
& =I(A ; C \mid B)+I(A ; D \mid B, C)=0 .
\end{aligned}
$$

This also shows that $I(A ; C \mid B, D)=0$. In addition, $I(A ; D \mid C)=0$, therefore,

$$
\begin{aligned}
I(A ; B \mid C, D) & =I(A ; B \mid C, D)+I(A ; D \mid C) \\
& =I(A ; B, D \mid C) \\
& =I(A ; B \mid C)+I(A ; D \mid B, C) \\
& =I(A ; B \mid C) .
\end{aligned}
$$

Equations (36), (37) and $I(A ; C \mid B, D)=0$ imply that

$$
I(A ; B \mid D)=I(A ; C \mid D)+I(A ; B \mid C, D)
$$

Thus, from (38)

$$
I(A ; B \mid D)=I(A ; C \mid D)+I(A ; B \mid C) \geq I(A ; B \mid C) .
$$

Proof of Lemma 4: We first prove that this lemma is true for $M=1$. By applying the chain rule twice to $I\left(X ; U_{1}, X+N_{1}\right)$, we obtain

$$
\begin{aligned}
& I\left(X ; X+N_{1} \mid U_{1}\right)-I\left(X ; U_{1} \mid X+N_{1}\right) \\
& \quad=I\left(X ; X+N_{1}\right)-I\left(X ; U_{1}\right) \\
& \quad=I\left(X ; X+N_{1}\right)-R\left(D_{1}\right) \geq 0 .
\end{aligned}
$$

Thus, $I\left(X ; U_{1} \mid X+N_{1}\right) \leq I\left(X ; X+N_{1} \mid U_{1}\right) \leq 1 / 2$ by the argument from steps (32)-(35).

Now suppose that this lemma holds for $M=k \geq 1$, i.e.,

$$
I\left(X ; U_{1}, U_{2}^{\prime}, \ldots, U_{k}^{\prime} \mid X+N_{k}\right) \leq k / 2
$$

where $N_{k} \sim \mathcal{N}\left(0, D_{k}\right)$ and $N_{k} \Perp\left(X, U_{1}, U_{2}^{\prime}, \ldots, U_{k}^{\prime}\right)$. Then for $M=k+1$, let $N_{k+1} \sim \mathcal{N}\left(0, D_{k+1}\right), N_{k}^{\prime} \Perp N_{k+1}, N_{k}^{\prime} \sim$ $\mathcal{N}\left(0, D_{k}-D_{k+1}\right)$, and

$$
\left(N_{k}^{\prime}, N_{k+1}\right) \Perp\left(X, U_{1}, U_{2}^{\prime}, \ldots, U_{k+1}^{\prime}\right) .
$$

By the chain rule

$$
\begin{aligned}
I(X ; & \left.U_{1}, U_{2}^{\prime}, \ldots, U_{k+1}^{\prime} \mid X+N_{k+1}\right) \\
= & I\left(X ; U_{1}, U_{2}^{\prime}, \ldots, U_{k}^{\prime} \mid X+N_{k+1}\right) \\
& +I\left(X ; U_{k+1}^{\prime} \mid U_{1}, U_{2}^{\prime}, \ldots, U_{k}^{\prime}, X+N_{k+1}\right) .
\end{aligned}
$$

We bound the first term on the right-hand side of (40) as

$$
\begin{aligned}
& I\left(X ; U_{1}, U_{2}^{\prime}, \ldots, U_{k}^{\prime} \mid X+N_{k+1}\right) \\
& \quad \leq I\left(X ; U_{1}, U_{2}^{\prime}, \ldots, U_{k}^{\prime} \mid X+N_{k+1}+N_{k}^{\prime}\right) \\
& \quad=I\left(X ; U_{1}, U_{2}^{\prime}, \ldots, U_{k}^{\prime} \mid X+N_{k}\right) \\
& \quad \leq k / 2
\end{aligned}
$$

where (41) follow from Lemma 3, and (42) follows from the previous assumption (39).
The second term on the right-hand side of (40) can be bounded as follows:

$$
\begin{aligned}
I & \left(X ; U_{k+1}^{\prime} \mid U_{1}, U_{2}^{\prime}, \ldots, U_{k}^{\prime}, X+N_{k+1}\right) \\
& \leq I\left(X ; X+N_{k+1} \mid U_{1}, U_{2}^{\prime}, \ldots, U_{k}^{\prime}, U_{k+1}^{\prime}\right) \\
& \quad=I\left(X-U_{k+1}^{\prime} ; X-U_{k+1}^{\prime}+N_{k+1} \mid U_{1}, U_{2}^{\prime}, \ldots, U_{k+1}^{\prime}\right) \\
& \leq I\left(X-U_{k+1}^{\prime} ; X-U_{k+1}^{\prime}+N_{k+1}\right) \\
& \leq 1 / 2
\end{aligned}
$$

where (43) follows by applying the chain rule twice to $I\left(X ; U_{1}, U_{2}^{\prime}, \ldots, U_{k}^{\prime}, U_{k+1}^{\prime}, X+N_{k+1}\right)$ to get

$$
\begin{aligned}
I(X ; & \left.X+N_{k+1} \mid U_{1}, U_{2}^{\prime}, \ldots, U_{k}^{\prime}, U_{k+1}^{\prime}\right) \\
& -I\left(X ; U_{k+1}^{\prime} \mid U_{1}, U_{2}^{\prime}, \ldots, U_{k}^{\prime}, X+N_{k+1}\right) \\
= & I\left(X ; U_{1}, U_{2}^{\prime}, \ldots, U_{k}^{\prime}, X+N_{k+1}\right) \\
& -I\left(X ; U_{1}, U_{2}^{\prime}, \ldots, U_{k}^{\prime}, U_{k+1}^{\prime}\right) \geq 0
\end{aligned}
$$

(44) follows since

$\left(U_{1}, U_{2}^{\prime}, \ldots, U_{k}^{\prime}, U_{k+1}^{\prime}\right) \rightarrow X-U_{k+1}^{\prime} \rightarrow X-U_{k+1}^{\prime}+N_{k+1}$

forms a Markov chain, and (45) parallels the chain of inequalities in steps (34)-(35).

Combining (40), (42), and (45) proves this lemma for $M=$ $k+1$. Finally, by induction, this lemma holds for any $M$.

Proof of Lemma 5: Let

$$
a=\frac{\sigma^{2}-D_{1}}{\sigma^{2}-D_{0}} \quad \text { and } \quad b=\frac{\sigma^{2}-D_{2}}{\sigma^{2}-D_{0}}
$$

then $D_{1}=a D_{0}+(1-a) \sigma^{2}$. From the convexity of the ratedistortion function

$$
R\left(D_{1}\right) \leq a R\left(D_{0}\right)+(1-a) R\left(\sigma^{2}\right)=a R\left(D_{0}\right) .
$$

By symmetry, $R\left(D_{2}\right) \leq b R\left(D_{0}\right)$, thus,

$$
\begin{aligned}
R\left(D_{1}\right)+R\left(D_{2}\right)-R\left(D_{0}\right) & \leq a R\left(D_{0}\right)+b R\left(D_{0}\right)-R\left(D_{0}\right) \\
& =\frac{\sigma^{2}+D_{0}-D_{1}-D_{2}}{\sigma^{2}-D_{0}} R\left(D_{0}\right)
\end{aligned}
$$

which is nonpositive if $D_{1}+D_{2}-D_{0} \geq \sigma^{2}$.

\section{ACKNOWLEDGMENT}

The authors are grateful to the Associate Editor and anonymous reviewers for their detailed suggestions and comments; in particular, Lemma 3 and the current improved result in Theorem 10 result from their insights.

\section{REFERENCES}

[1] E. A. Riskin and R. M. Gray, "A greedy tree growing algorithm for the design of variable rate vector quantizers," IEEE Trans. Signal Processing, vol. 39, pp. 2500-2507, Nov. 1991.

[2] W. B. Pennebaker and J. L. Mitchell, JPEG Still Image Data Compression Standard. New York: Van Nostrand Reinhold, 1993.

[3] J. M. Shapiro, "Embedded image coding using zerotrees of wavelet coefficients," IEEE Trans. Signal Processing, vol. 41, pp. 3445-3462, Dec. 1993.

[4] A. Said and W. A. Pearlman, "A new, fast, and efficient image codec based on set partitioning in hierarchical trees," IEEE Trans. Circuits Syst. Video Technol., vol. 6, pp. 243-250, June 1996.

[5] M. Effros, "Practical multiresolution source coding: TSVQ revisited.," in Proc. IEEE Data Compression Conf., Snowbird, UT, Mar. 1998, pp. 53-62.

[6] M. Effros and D. Dugatkin, "Multiresolution vector quantization.," IEEE Trans. Inform. Theory, submitted for publication. 
[7] V. Koshelev, "Hierarchical coding of discrete sources," Probl. Pered. Inform., vol. 16, no. 3, pp. 31-49, 1980.

[8] W. H. R. Equitz and T. M. Cover, "Successive refinement of information," IEEE Trans. Inform. Theory, vol. 37, pp. 269-275, Mar. 1991.

[9] L. Lastras and T. Berger, "On the refinement of the binary symmetric Markov source," in Proc. IEEE Int. Symp. Information Theory, Washington, DC, June 2001, p. 195.

[10] J. Chow and T. Berger, "Failure of successive refinement for symmetric Gaussian mixtures," IEEE Trans. Inform. Theory, vol. 43, pp. 350-352, Jan. 1997.

[11] B. Rimoldi, "Successive refinement of information: Characterization of achievable rates," IEEE Trans. Inform. Theory, vol. 40, pp. 253-259, Jan. 1994.

[12] M. Effros, "Distortion-rate bounds for fixed- and variable-rate multiresolution source codes," IEEE Trans. Inform. Theory, vol. 45, pp 1887-1910, Sept. 1999.

[13] L. Lastras and T. Berger, "All sources are nearly successively refinable," IEEE Trans. Inform. Theory, vol. 47, pp. 918-926, Mar. 2001.

[14] R. Zamir, "The rate loss in the Wyner-Ziv problem," IEEE Trans. Inform. Theory, vol. 42, pp. 2073-2084, Nov. 1996.

[15] E. Tuncel and K. Rose, "Additive successive refinement," in Proc. IEEE Int. Symp. Information Theory, Washington, DC, June 2001, p. 31.
[16] — - "Additive successive refinement," IEEE Trans. Inform. Theory, submitted for publication

[17] A. A. El Gamal and T. M. Cover, "Achievable rates for multiple descriptions," IEEE Trans. Inform. Theory, vol. IT-28, pp. 851-857, Nov. 1982

[18] E. Tuncel and K. Rose, private communication, Mar. 2002.

[19] T. M. Cover and J. A. Thomas, Elements of Information Theory. New York: Wiley, 1991.

[20] A. Buzo, A. H. Gray, Jr, R. M. Gray, and J. D. Markel, "Speech coding based upon vector quantization," IEEE Trans. Acoust., Speech, Signal Processing, vol. ASSP-28, pp. 562-574, Oct. 1980.

[21] E. A. Riskin, R. Ladner, R. Y. Wang, and L. E. Atlas, "Index assignment for progressive transmission of full-search vector quantization," IEEE Trans. Image Processing, vol. 3, pp. 307-312, May 1994.

[22] J. Ziv, "On universal quantization," IEEE Trans. Inform. Theory, vol. IT-31, pp. 344-347, May 1985.

[23] R. Zamir and M. Feder, "On lattice quantization noise," IEEE Trans. Inform. Theory, vol. 42, pp. 1152-1159, July 1996.

[24] Y. Frank-Dayan and R. Zamir, "Dithered lattice-based quantizers for multiple descriptions," IEEE Trans. Inform. Theory, vol. 48, pp. 192-204, Jan. 2002.

[25] H. Feng, Q. Zhao, and M. Effros, "Network source coding using entropy constrained dithered quantization," in Proc. IEEE Data Compression Conf., Snowbird, UT, Mar. 2003. 